\title{
Robust Adaptive Fractional Fast Terminal Sliding Mode Controller for Microgyroscope
}

\author{
Juntao Fei $(\mathbb{D}$, Zhe Wang, and Xiao Liang \\ College of IoT Engineering, Jiangsu Key Laboratory of Power Transmission and Distribution Equipment Technology, \\ Hohai University, Changzhou 213022, China
}

Correspondence should be addressed to Juntao Fei; jtfei@yahoo.com

Received 24 February 2020; Revised 5 June 2020; Accepted 29 June 2020; Published 23 July 2020

Academic Editor: Yan-Ling Wei

Copyright (c) 2020 Juntao Fei et al. This is an open access article distributed under the Creative Commons Attribution License, which permits unrestricted use, distribution, and reproduction in any medium, provided the original work is properly cited.

In this paper, a robust adaptive fractional fast terminal sliding mode controller is introduced into the microgyroscope for accurate trajectory tracking control. A new fast terminal switching manifold is defined to ensure fast finite convergence of the system states, where a fractional-order differentiation term emerges into terminal sliding surface, which additionally generates an extra degree of freedom and leads to better performance. Adaptive algorithm is applied to estimate the damping and stiffness coefficients, angular velocity, and the upper bound of the lumped nonlinearities. Numerical simulations are presented to exhibit the validity of the proposed method, and the comparison with the other two methods illustrates its superiority.

\section{Introduction}

As an important measuring component, microgyroscope is widely used in military and civil fields with low cost and high reliability. In recent years, many researchers have endeavored to study advanced control technologies applied to microgyroscopes like adaptive control, sliding mode control, and intelligent control. Adaptive mechanism is an efficacious control scheme for various nonlinear systems due to its abilities to dynamically estimate system parameters. Ma and Ghasemi-Nejhad [1] introduced an adaptive fuzzy logic control strategy into the flexible active composite manipulator for vibration suppression. Sanner and Slotine [2] and Li et al. [3] designed adaptive fuzzy and neural methods for nonlinear dynamic systems. In $[4,5]$, a robust adaptive control law incorporating the projection algorithm is utilized to counteract time-varying unknown bounded environmental disturbances of ship system. In [6], an adaptive control scheme was proposed to achieve the goal of trajectory tracking for robot manipulators with uncertain dynamics and kinematics. In [7], an adaptive scheme was combined with $\mathrm{H}$-infinite control technique to obtain high tracking performance of robotic manipulators. Fuzzy control and neural network control methods were developed for uncertain nonlinear dynamic systems in [8-13]. Adaptive control strategies to compensate for fabrication imperfects were proposed for a microgyroscope in [14-16].

Sliding mode control (SMC) is a useful and efficient control method for nonlinear systems due to its robustness and simplicity properties. Sliding mode control and observation were investigated for complex industrial systems in [17]. An adaptive algorithm was incorporated into sliding controller using neural controller to improve the performance of a microgyroscope in [18]. An SMC was proposed for an uncertain discrete singular system with time-varying delays and external disturbances in [19]. Lu et al. [20] proposed an improved second-order sliding control strategy for nonlinear underactuated systems. Sliding mode controller and fuzzy and neural network controller for active power filters were investigated in [21-26]. A continuous SMC with adaptive algorithm to estimate the upper bound of the uncertainties was proposed in [27], which could alleviate the chattering without reducing accuracy. Apart from chattering, another deficiency of conventional SMC is that the linear sliding surface can only ensure the asymptotic stability but not in finite-time convergence. To overcome this problem, a fast terminal sliding mode control (TSMC) with nonlinear switching hyperplanes was proposed in [28] 
to ensure fast finite time of convergence compared to conventional SMC. In [29], a TSMC method was investigated for permanent magnet synchronous motor servo speed regulation system, which guaranteed that the system states converge to the equilibrium point in a finite time. In [30], an adaptive TSMC was synthesized into the robotic manipulators with time-delay control to improve the response characteristics of the system and attenuate the uncertainties. In [31-33], an adaptive fast TSMC and adaptive second-order fast NTSM are, respectively, synthesized into the autonomous underwater vehicles control system, which can reach local exponential convergence with zero position tracking error. In $[34,35]$, a time-delay estimation (TDE) technique combined with nonsingular fast TSMC is synthesized into cable-driven manipulators to realize accurate tracking control. A fast TSMC was applied to a Van der Pol oscillator with an integral filter, which ensured a finite-time convergence to zero dynamics in [36].

SMC can also be combined with fractional calculus for better control performance [37-39]. An adaptive SMC scheme was proposed for a fractional-order nonlinear system in the presence of uncertainties in [40]. In [41], a fractional-order TSMC is incorporated with adaptive scheme to deal with uncertain dynamics of exoskeleton under unknown external disturbances. In [42], an adaptive fractional-order TSMC was proposed for robot manipulators with uncertainties and external disturbances, which achieved better tracking performance and finite-time convergence. Nojavanzadeh and Badamchizadeh [43] proposed an adaptive fractional TSMC method with a fractional-order integral sliding surface of linear motor. A new compound fractional-order terminal sliding mode control for a microgyroscope was proposed in [44]. In [45], a novel discrete time fractional-order sliding control strategy was derived for a linear motor system, which guaranteed the desired tracking performance. In [46], the fractional order (FO) with nonsingular fast TSMC is adopted for the tracking of the lower-limb rehabilitation exoskeleton. In $[47,48]$, a fractional-order nonsingular TSMC combined with adaptive time-delay control is introduced into adaptive timedelay control to guarantee accurate trajectory tracking of cabledriven robots.

In this paper, motivated by the discussion above, an adaptive fractional fast terminal sliding mode control strategy is proposed for a microgyroscope. The main contributions are emphasized in the following expressions:

(1) A new form of fast terminal sliding surface is defined, which not only guarantees the fast finite-time convergence of system states to equilibrium point but also relaxes the restriction on parameter selection. It solved the problem that the linear sliding surface could only guarantee that the convergence is asymptotical but not in finite time.

(2) Furthermore, the superior characteristic of the proposed method is that a fractional-order term is incorporated into sliding phase, which offers an extra degree of freedom fractional order and flexible control laws for designers to achieve better performance compared to integer one.
(3) Based on fractional-order fast TSMC scheme, an adaptive method is incorporated to dynamically estimate both the system parameters and the upper bound of the lumped nonlinearities. In addition, continuous control inputs are designed to deal with undesirable chattering phenomenon.

(4) Compared to fractional-order SMC and integerorder fast TSMC, it can be seen that fractional technique can improve the accuracy of the closedloop feedback system and fast terminal sliding mode control can provide shorter convergence time.

\section{Dynamics of Microgyroscope}

A $z$-axis microgyroscope system is described in Figure 1. Referring to [17], in order to derive the model of the microgyroscope, some assumptions have been made: (1) the gyroscope moves with a constant linear speed and rotates at a constant angular velocity; (2) the microgyroscope undergoes rotations only in $x$-axis and $y$-axis; and (3) the centrifugal forces are negligible. Then, the dynamics of the microgyroscope are described as follows:

$$
\begin{aligned}
& m \ddot{x}+d_{x} \dot{x}+\left[k_{x}-m\left(\Omega_{y}^{2}+\Omega_{z}^{2}\right)\right] x+m \Omega_{x} m \Omega_{y} y=u_{x}+2 m \Omega_{z} \dot{y}, \\
& m \ddot{x}+d_{y} \dot{y}+\left[k_{y}-m\left(\Omega_{x}^{2}+\Omega_{z}^{2}\right)\right] y+m \Omega_{x} m \Omega_{y} x=u_{y}-2 m \Omega_{z} \dot{x},
\end{aligned}
$$

where $m$ is the mass of proof mass, $d_{x, y}, k_{x, y}$ are the damping and spring coefficients, $\Omega_{x, y, z}$ are the angular velocity and $u_{x, y}$ are the control inputs, and $2 m \Omega_{z} \dot{x}$ and $2 m \Omega_{z} \dot{y}$ are the terms used to measure the angular rate $\Omega_{z}$, which are due to the Coriolis forces.

As shown in (1), in an ideal gyroscope, only the component of the angular rate along the $z$-axis $\Omega_{z}$ causes a dynamic coupling between the $x$-axis and $y$-axis under the assumption that $\Omega_{x, y} \approx \Omega_{x} \Omega_{y} \approx 0$. In practice, however, small fabrication imperfections always occur, which cause dynamic coupling between the $x$-axis and $y$-axis through the asymmetric spring and damping terms. By taking into account the fabrication imperfections, the dynamic equations (1) are modified as follows [49].

$$
\begin{aligned}
& m \ddot{x}+d_{x x} \dot{x}+d_{x y} \dot{y}+k_{x x} x+k_{x y} y=u_{x}+2 m \Omega_{z} \dot{y}, \\
& m \ddot{y}+d_{x y} \dot{x}+d_{y y} \dot{y}+k_{x y} x+k_{y y} y=u_{y}-2 m \Omega_{z} \dot{x},
\end{aligned}
$$

where $k_{x x}, k_{y y}$ and $d_{x x}, d_{y y}$ denote the spring coefficients terms and damping terms, respectively. The fabrication imperfections mainly contribute to the asymmetric spring and damping terms $k_{x y}$ and $d_{x y}$. Therefore, these terms are unknown but can be assumed to be small. The $x$-axis and $y$-axis spring and damping terms are mostly known but have unknown variations from their nominal values. The proof mass can be determined very accurately. The components of angular rate along the $x$-axis and $y$-axis are absorbed as part of the spring terms as unknown variations. Note that the spring coefficients $k_{x x}$ and $k_{y y}$ also include the electrostatic spring softness.

Based on a reference mass $m$, length $q_{0}$, and natural resonance frequency $\omega_{0}$, the nondimensionalization of equation (2) can be derived as 


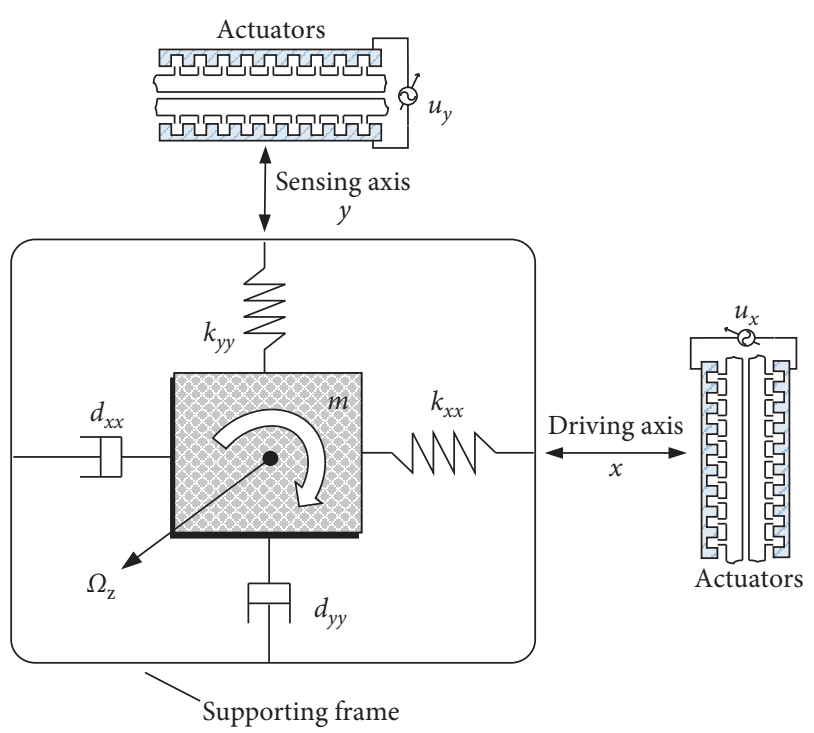

FIGURE 1: Schematic structure of a $z$-axis microgyroscope.

$$
\frac{\ddot{q}}{q_{0}}+\frac{D}{m \omega_{0}} \frac{\dot{q}}{q_{0}}+\frac{K}{m \omega_{0}^{2}} \frac{q}{q_{0}}=\frac{u}{m \omega_{0}^{2} q_{0}}-2 \frac{\Omega_{z}}{\omega_{0}} \frac{\dot{q}}{q_{0}} .
$$

Define a set of new parameters as follows:

$$
\begin{aligned}
& q^{*}=\frac{q}{q_{0}}, \\
& d_{x y}^{*}=\frac{d_{x, y}}{m \omega_{0}}, \\
& \Omega_{z}^{*}=\frac{\Omega_{z}}{\omega_{0}}, \\
& u_{x}^{*}=\frac{u_{x}}{m \omega_{0}^{2} q_{0}}, \\
& u_{y}^{*}=\frac{u_{y}}{m \omega_{0}^{2} q_{0}}, \\
& \omega_{x}=\sqrt{\frac{k_{x x}}{m \omega_{0}^{2}}}, \\
& \omega_{y}=\sqrt{\frac{k_{y y}}{m \omega_{0}^{2}}}, \\
& \omega_{x y}
\end{aligned}
$$

By equivalent transformation and ignoring the superscript $\left({ }^{*}\right)$ to clarify notation, the nondimensional representation of the microgyroscope can be depicted in the following expression:

$$
\ddot{q}+D \dot{q}+K q=u-2 \Omega \dot{q},
$$

where

$$
\begin{aligned}
q & =\left[\begin{array}{l}
x \\
y
\end{array}\right], \\
D & =\left[\begin{array}{ll}
d_{x x} & d_{x y} \\
d_{x y} & d_{y y}
\end{array}\right], \\
K & =\left[\begin{array}{ll}
\omega_{x}^{2} & \omega_{x y} \\
\omega_{x y} & \omega_{y}^{2}
\end{array}\right], \\
u & =\left[\begin{array}{ll}
u_{x} \\
u_{y}
\end{array}\right], \\
\Omega & =\left[\begin{array}{cc}
0 & -\Omega_{z} \\
\Omega_{z} & 0
\end{array}\right] .
\end{aligned}
$$

\section{Fractional Fast Terminal Sliding Mode Control}

3.1. Preliminary Introduction of Fractional Order. As the generalization concept of the conventional differentiation and integration, Caputo (C), Grunwald-Letnikov (GL), and Riemann-Liouville (RL) are the three main commonly used definitions in engineering, science, and economics fields [50], especially the Caputo definition, since its initial conditions are of the same form as those for integer-order differential equations. Besides, considering its wide application and well-understood physical sense, the following Caputo fractional derivative of order $\alpha$ of a continuous function $f(x)$ is given by [51]:

$$
{ }_{a} D_{t}^{\alpha} f(t)= \begin{cases}\frac{1}{\Gamma(n-\alpha)} \int_{a}^{t} \frac{f^{(n)}(\tau)}{(t-\tau)^{\alpha-n+1}} \mathrm{~d} \tau, & n-1<\alpha<n, \\ \frac{\mathrm{d}^{n}}{\mathrm{~d} t^{n}} f(t), & \alpha=n,\end{cases}
$$

where $\Gamma(\cdot)$ is Gamma function that satisfies

$$
\Gamma(\gamma)=\int_{0}^{\infty} e^{-t} t^{\gamma-1} \mathrm{~d} t
$$

For simplifying the notation, the fractional derivative order $\alpha$ with the lower bound at 0 is expressed as $D^{\alpha}$ instead of ${ }_{0} D_{t}^{\alpha}$.

It is noted that if $\alpha=0$, the operation $D^{0} f(x)$ satisfies $D^{0} f(x)=f(x)$.

3.2. Fractional Fast Terminal Sliding Mode Control. The dynamics of MEMS gyroscope with parameter variations and external disturbances can be described as

$$
\ddot{q}+(D+2 \Omega+\Delta D) \dot{q}+(K+\Delta K) q=u+d,
$$

where $\Delta D$ and $\Delta K$ are the unknown variations from their nominal values of $x$-axis and $y$-axis spring and damping terms. $d$ denotes an external disturbance.

Define the system lumped uncertainty as 


$$
f=d-\Delta D \dot{q}-\Delta K q .
$$

We assume that the unknown parameter terms and external disturbance $\Delta D, \Delta K$, and $d$ are bounded such that

$$
\begin{aligned}
& |d| \leq b_{0}, \\
& |K| \leq b_{1}, \\
& |D| \leq b_{2},
\end{aligned}
$$

where $b_{0}, b_{1}, b_{2}$ are the unknown positive constants.

According to the above assumption, the following inequality holds:

$$
\|f\| \leq b_{0}+b_{1}\|q\|+b_{2}\|\dot{q}\| .
$$

Accordingly, one can rewrite the dynamics of system (9) as

$$
\ddot{q}+(D+2 \Omega) \dot{q}+K q=u+f .
$$

The uncertain system (13) is expected to follow a reference trajectory in finite time. Assume that $q$ and $q_{r}$ are actual and desired positions, respectively; then the tracking error and its time derivative can be defined as

$$
\begin{aligned}
& e=q-q_{r}, \\
& \dot{e}=\dot{q}-\dot{q}_{r} .
\end{aligned}
$$

A traditional integer-order fast terminal sliding mode (TSM) surface is expressed as in [38]:

$$
s=\dot{e}+\alpha e+\beta e^{q / p},
$$

where $\alpha=\operatorname{diag}\left(\alpha_{1}, \alpha_{2}\right), \beta=\operatorname{diag}\left(\beta_{1}, \beta_{2}\right)$ are constant matrices and $p, q$ are odd positive integers and satisfy $p>q>0$. However, the fractional power $q / p$ may cause the term $e^{q / p} \notin R$ when $e<0$, which means $\dot{e} \notin R$ contradicting with the system we have considered. So, a modified fractional fast terminal sliding surface is proposed as

$$
s=\dot{e}+\lambda_{1} D^{\alpha-1} e+\lambda_{2}|e|^{\gamma} \operatorname{sign}(e)
$$

where $\lambda_{1}, \lambda_{2}$ are positive diagonal matrices, $\alpha$ is FO within $0<\alpha<1$, and $\gamma$ is a positive constant with range within $0<\gamma<1$.

Remark 1. The singularity problem in (16) can be avoided by nonsingular terminal sliding surface.

Then, its time derivative is

$$
\begin{aligned}
\dot{s} & =\ddot{e}+\lambda_{1} D^{\alpha} e+\lambda_{2} \gamma \operatorname{diag}\left(|e|^{\gamma-1}\right) \dot{e} \\
& =u+f-(D+2 \Omega) \dot{q}-K q-\ddot{q}_{r}+\lambda_{1} D^{\alpha} e+\lambda_{2} \gamma \operatorname{diag}\left(|e|^{\gamma-1}\right) \dot{e} .
\end{aligned}
$$

The control torque law based on fractional fast TSM is designed as follows:

$$
u_{e q}=(D+2 \Omega) \dot{q}+K q+\ddot{q}_{r}-\lambda_{1} D^{\alpha} e-\lambda_{2} \gamma \operatorname{diag}\left(|e|^{\gamma-1}\right) \dot{e} .
$$

The control law (18) requires that $f$ should be known, which is impossible in practice. Considering that the bound of uncertainties is $\|f\| \leq b_{0}+b_{1}\|q\|+b_{2}\|\dot{q}\|$, we introduce the discontinuous term $\Delta u$ to compensate for the lumped uncertainty as

$$
\Delta u=-\frac{s}{\|s\|}\left(b_{0}+b_{1}\|q\|+b_{2}\|\dot{q}\|\right) .
$$

Then the fractional fast terminal sliding controller applied to the microgyroscope can be rewritten as

$$
\begin{aligned}
u^{\prime}= & u_{\mathrm{eq}}+\Delta u \\
= & (D+2 \Omega) \dot{q}+K q+\ddot{q}_{r}-\lambda_{1} D^{\alpha} e-\lambda_{2} \gamma \operatorname{diag}\left(|e|^{\gamma-1}\right) \dot{e} \\
& -\frac{s}{\|s\|}\left(b_{0}+b_{1}\|q\|+b_{2}\|\dot{q}\|\right) .
\end{aligned}
$$

Select a Lyapunov function $V_{1}$ as

$$
V_{1}=\frac{1}{2} s^{T} s
$$

Differentiating (21) and then substituting equations (10), (17), and (20) into it, we get

$$
\begin{aligned}
\dot{V}_{1} & =s^{T}\left(f-\frac{s}{\|s\|}\left(b_{0}+b_{1}\|q\|+b_{2}\|\dot{q}\|\right)\right) \\
& \leq\|s\|\left(\|f\|-\left(b_{0}+b_{1}\|q\|+b_{2}\|\dot{q}\|\right)\right) \\
& \leq-\min \left(\left(b_{0}+b_{1}\|q\|+b_{2}\|\dot{q}\|\right)-\|f\|\right)\|s\| \\
& \leq-\varepsilon\|s\| \\
& \leq-\varepsilon V_{1}^{1 / 2},
\end{aligned}
$$

where $\varepsilon=\min \left(\left(b_{0}+b_{1}\|q\|+b_{2}\|\dot{q}\|\right)-\|f\|\right)>0$.

Then, (22) can be rewritten as

$$
\frac{\mathrm{d} V_{1}}{\sqrt{V_{1}}} \leq-\sqrt{2} \varepsilon \mathrm{d} t
$$

Denoting $t_{r}$ as the total time from the initial state to $\dot{s}=0$, taking the integral of (23) with respect to time, we obtain

$$
\int_{V_{1}(0)}^{V_{1}\left(t_{r}\right)} \frac{\mathrm{d} V_{1}}{\sqrt{V_{1}}} \leq-\sqrt{2} \int_{0}^{t_{r}} \varepsilon \mathrm{d} t .
$$

Then, from (24), the fractional fast TSMC manifold will be attained in a finite time $t_{r}$, which satisfies

$$
t_{r} \leq \frac{\sqrt{2\left(V_{1}(0)-V_{1}\left(t_{r}\right)\right)}}{\varepsilon} .
$$

Remark 2. To prove that it can converge in finite time, a stopping time is defined as follows:

$$
t_{s}=\inf \left\{t \geq t_{r}: e(t)=0\right\},
$$

where $t_{r}$ denotes the reaching time. Then it will be proved that there exists $t_{d} \in\left[t_{r}, \infty\right)$, so that $t_{s}<t_{d}$. 
Considering the fractional integral and derivative operators, using equation (16) yields

$$
\begin{aligned}
& D^{-2}\left(D^{2} e(t)\right) \\
& =-\lambda_{1} D^{\alpha-2} e(t)-D^{-1}\left(\lambda_{2}|e(t)|^{\gamma} \operatorname{sign}(e(t))\right. \\
& \left.\quad-\lambda_{2}\left|e\left(t_{r}\right)\right|^{\gamma} \operatorname{sign}\left(e\left(t_{r}\right)\right)\right) .
\end{aligned}
$$

Referring to [50], one can obtain that

$$
\begin{aligned}
& e(t)-\left[{ }_{t r} D_{t} e(t)\right]_{t=t_{r}} \frac{t-t_{r}}{2}-[e(t)]_{t=t_{r}}=\lambda_{1} D^{\alpha-2} e(t) \\
+ & D^{-1}\left(\lambda_{2}|e(t)|^{\gamma} \operatorname{sign}(e(t))-\lambda_{2}\left|e\left(t_{r}\right)\right|^{\gamma} \operatorname{sign}\left(e\left(t_{r}\right)\right)\right) .
\end{aligned}
$$

Define $C=\lambda_{2}\left|e\left(t_{r}\right)\right|^{\gamma} \operatorname{sign}\left(e\left(t_{r}\right)\right)$.

According to [51], we can get

$$
\begin{aligned}
& \lambda_{1} D^{\alpha-2} e(t)+D^{-1}\left(\lambda_{2}|e(t)|^{\gamma} \operatorname{sign}(e(t))-C\right) \\
& \leq K_{1}\left\|\lambda_{1} e(t)\right\|+K_{2}\left\|\lambda_{2}|e(t)|^{\gamma} \operatorname{sign}(e(t))-C\right\| .
\end{aligned}
$$

Then

$$
\begin{aligned}
& \left\|e(t)-\left[{ }_{t r} D_{t} e(t)\right]_{t=t_{r}} \frac{t-t_{r}}{2}\right\|-\left\|[e(t)]_{t=t_{r}}\right\| \\
& \leq K_{1}\left\|\lambda_{1} e(t)\right\|+K_{2}\left\|\lambda_{2}|e(t)|^{\gamma} \operatorname{sign}(e(t))-C\right\| .
\end{aligned}
$$

Note that $e\left(t_{d}\right)=0$ and $\dot{e}\left(t_{d}\right)=0$ at $t=t_{d}$, which yield

$$
\left\|\left[\frac{{ }_{t r} D_{t} e(t)}{2}\right]_{t=t_{r}}\right\|\left(t_{d}-t_{r}\right) \leq\left\|[e(t)]_{t=t_{r}}\right\|+K_{2}\|C\| .
$$

Then, we have

$$
t_{d} \leq \frac{2\left(\left\|e\left(t_{r}\right)\right\|+K_{2}\|C\|\right)}{\dot{e}\left(t_{r}\right)}+t_{r} .
$$

Thus, the proposed controller guarantees the finite-time stability.

Remark 3. The discontinuous control $\Delta u$ will bring the chattering phenomenon, which is undesirable in the control procedure, so $\Delta u$ is replaced by the following expression in the actual controller design:

$$
\Delta u_{1}= \begin{cases}-\frac{s}{\|s\|}\left(b_{0}+b_{1}\|q\|+b_{2}\|\dot{q}\|\right), & \|s\| \geq \delta, \\ -\frac{s}{\delta^{2}}\left(\|s\|\left(b_{0}+b_{1}\|q\|+b_{2}\|\dot{q}\|\right)\right), & \|s\|<\delta,\end{cases}
$$

where $\delta>0$ is the size of dead zone.

Then the controller applied to the microgyroscope is designed as

$$
u^{\prime}=u_{\text {eq }}+\Delta u_{1} .
$$

It should be noted that the continuous fast TSM control attenuates chattering effect at the expense of robustness properties, and the upper bound of the lumped uncertainties should be known.

\section{Robust Adaptive Fractional Fast Terminal Sliding Mode Control}

Prior knowledge of the upper bound of the lumped uncertainties is necessary when applying the TSM control to the microgyroscope. However, it is difficult to determine this bound beforehand for the unpredictability and complexity of the structure. The larger $\delta$ selected to eliminate the chattering problem may generate the loss in robustness. Besides, in previous step, the control law (34) is developed in the case of available parameter variations $D, K, \Omega$ which are unknown in practice systems. So, a robust adaptive fractional-order TSM control strategy is designed for the microgyroscope described by equation (13) to solve the problems above, and the block diagram of the designed controller is depicted in Figure 2.

Adaptive control is integrated to estimate the upper bound of the lumped uncertainties $b_{0}, b_{1}, b_{2}$ with $\widehat{b}_{0}, \vec{b}_{1}, \widehat{b}_{2}$ and to estimate the system parameters $D, K, \Omega$ with $\widehat{D}, \widehat{K}, \widehat{\Omega}$, respectively. Then the control law (34) can be modified as

$$
u=(\widehat{D}+2 \widehat{\Omega}) \dot{q}+\widehat{K} q+\ddot{q}_{r}-\lambda_{1} D^{\alpha} e-\lambda_{2} \gamma \operatorname{diag}\left(|e|^{\gamma-1}\right) \dot{e}+\Delta u_{2},
$$

where $\Delta u_{2}$ is described as

$$
\Delta u_{2}=-\frac{s}{\|s\|}\left(\widehat{b}_{0}+\widehat{b}_{1}\|q\|+\widehat{b}_{2}\|\dot{q}\|\right) .
$$

Define the parameter estimation error as

$$
\begin{aligned}
& \widetilde{D}=\widehat{D}-D, \\
& \widetilde{K}=\widehat{K}-K, \\
& \widetilde{\Omega}=\widehat{\Omega}-\Omega, \\
& \widetilde{b}_{i}=b_{i}-\widehat{b}_{i}, \quad(i=0,1,2) .
\end{aligned}
$$

Substituting the control law (35) into equation (17) results in

$$
\dot{s}=(\widetilde{D}+2 \widetilde{\Omega}) \dot{q}+\widetilde{K} q+f-\frac{s}{\|s\|}\left(\widehat{b}_{0}+\widehat{b}_{1}\|q\|+\widehat{b}_{2}\|\dot{q}\|\right) .
$$

Define a Lyapunov function candidate as

$$
\begin{aligned}
V= & \frac{1}{2} s^{T} s+\frac{1}{2} \operatorname{tr}\left\{\widetilde{D} M^{-1} \widetilde{D}^{T}\right\}+\frac{1}{2} \operatorname{tr}\left\{\widetilde{K} N^{-1} \widetilde{K}^{T}\right\} \\
& +\frac{1}{2} \operatorname{tr}\left\{\widetilde{\Omega} P^{-1} \widetilde{\Omega}^{T}\right\}+\frac{1}{2} \sum_{i=0}^{2} x_{i}^{-1} \widetilde{b}_{i}^{2},
\end{aligned}
$$

where $M=M^{T}>0, N=N^{T}>0$, and $P=P^{T}>0$; they are all positive definite matrices and $\operatorname{tr}\{\cdot\}$ denotes the matrix trace operator.

Taking derivative of equation (39) with respect to time and then using (38) yield 


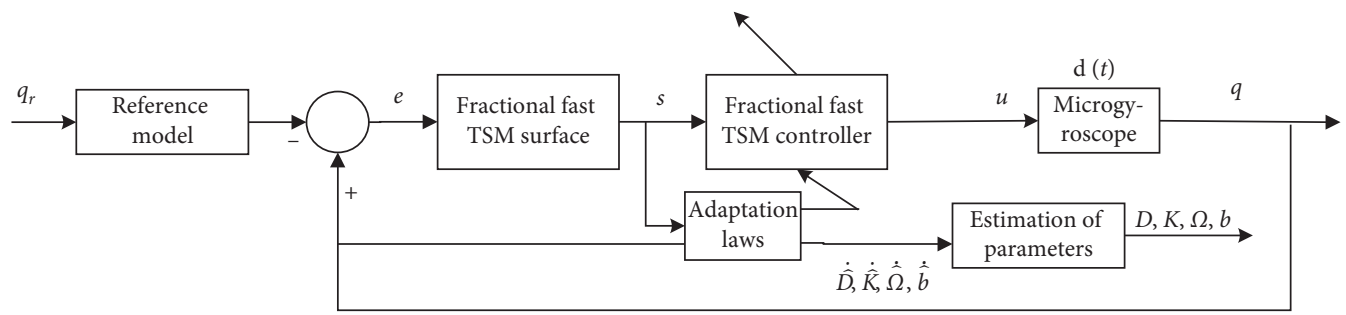

FIGURE 2: The block diagram of the robust adaptive fractional fast TSM controller.

$$
\begin{aligned}
\dot{V}= & s^{T} \dot{s}+\operatorname{tr}\left\{\widetilde{D} M^{-1} \dot{\tilde{D}}^{T}\right\}+\operatorname{tr}\left\{\widetilde{K} N^{-1} \dot{\tilde{K}}^{T}\right\}+\operatorname{tr}\left\{\widetilde{\Omega} P^{-1} \dot{\tilde{\Omega}}^{T}\right\}+\sum_{i=0}^{2} x_{i}^{-1} \widetilde{b}_{i} \dot{\vec{b}}_{i} \\
= & s^{T}\left((\widetilde{D}+2 \widetilde{\Omega}) \dot{q}+K q+f-\frac{s}{\|s\|}\left(\widehat{b}_{0}+\widehat{b}_{1}\|q\|+\widehat{b}_{2}\|\dot{q}\|\right)\right) \\
& +\operatorname{tr}\left\{\widetilde{D} M^{-1} \dot{\tilde{D}}^{T}\right\}+\operatorname{tr}\left\{\widetilde{K} N^{-1} \dot{\tilde{K}}^{T}\right\}+\operatorname{tr}\left\{\widetilde{\Omega} P^{-1} \dot{\tilde{\Omega}}^{T}\right\}+\sum_{i=0}^{2} x_{i}^{-1} \widetilde{b}_{i} \dot{\vec{b}}_{i} \\
= & s^{T} \widetilde{D} \dot{q}+\operatorname{tr}\left\{\widetilde{D} M^{-1} \dot{\tilde{D}}^{T}\right\}+s^{T} \widetilde{K} q+\operatorname{tr}\left\{\widetilde{K} N^{-1} \dot{\tilde{K}}^{T}\right\}+2 s^{T} \widetilde{\Omega} \dot{q} \\
& +\operatorname{tr}\left\{\widetilde{\Omega} P^{-1} \dot{\widetilde{\Omega}}^{T}\right\}-\sum_{i=0}^{2} x_{i}^{-1} \widetilde{b}_{i} \dot{\dot{b}_{i}}+s^{T} f-\|s\|\left(\widehat{b}_{0}+\widehat{b}_{1}\|q\|+\widehat{b}_{2}\|\dot{q}\|\right) .
\end{aligned}
$$

Since $D=D^{T}, K=K^{T}, \Omega=-\Omega^{T}$ and $s^{T} \widetilde{D} \dot{q}=\dot{q}^{T} \widetilde{D} s$ are scalar, we have

$$
s^{T} \widetilde{D} \dot{q}=\frac{1}{2}\left(s^{T} \widetilde{D} \dot{q}+\dot{q}^{T} \widetilde{D} s\right)=\operatorname{tr}\left\{\frac{1}{2}\left(\widetilde{D} \dot{q} s^{T}+\widetilde{D} s \dot{q}^{T}\right)\right\} .
$$

Simultaneously, one can obtain

$$
\begin{array}{r}
s^{T} \widetilde{K} q=\frac{1}{2}\left(s^{T} \widetilde{K} q+q^{T} \widetilde{K} s\right)=\operatorname{tr}\left\{\frac{1}{2}\left(\widetilde{K} q s^{T}+\widetilde{K} s q^{T}\right)\right\}, \\
2 s^{T} \widetilde{\Omega} \dot{q}=\left(s^{T} \widetilde{\Omega} \dot{q}+\dot{q}^{T} \widetilde{\Omega} s\right)=\operatorname{tr}\left\{\left(\widetilde{\Omega} \dot{q} s^{T}+\widetilde{\Omega} s \dot{q}^{T}\right)\right\} .
\end{array}
$$

Substituting (41) and (42) into equation (40) yields

$$
\begin{aligned}
\dot{V} & =\operatorname{tr}\left\{\widetilde{D}\left(M^{-1} \dot{\tilde{D}}^{T}+\frac{1}{2}\left(\dot{q} s^{T}+s \dot{q}^{T}\right)\right)\right\} \\
& +\operatorname{tr}\left\{\widetilde{K}\left(N^{-1} \dot{\tilde{K}}^{T}+\frac{1}{2}\left(q s^{T}+s q^{T}\right)\right)\right\}+\operatorname{tr}\left\{\widetilde{\Omega}\left(P^{-1} \dot{\widetilde{\Omega}}^{T}+\dot{q} s^{T}+s \dot{q}^{T}\right)\right\} \\
& -\sum_{i=0}^{2} x_{i}^{-1} \widetilde{b}_{i} \dot{\vec{b}}_{i}+s^{T} f-\|s\|\left(\widehat{b}_{0}+\widehat{b}_{1}\|q\|+\widehat{b}_{2}\|\dot{q}\|\right) .
\end{aligned}
$$

In order to guarantee that $\dot{V} \leq 0$, the online adaptation laws for the parameters are designed as

$$
\begin{aligned}
& \dot{\tilde{D}}^{T}=\dot{\widehat{D}}^{T}=-\frac{1}{2} M\left(\dot{q} s^{T}+s \dot{q}^{T}\right), \\
& \dot{\tilde{K}}^{T}=\dot{\hat{K}}^{T}=-\frac{1}{2} N\left(q s^{T}+s q^{T}\right), \\
& \dot{\widetilde{\Omega}}^{T}=\dot{\widehat{\Omega}}^{T}=-P\left(\dot{q} s^{T}+s \dot{q}^{T}\right), \\
& \dot{\vec{b}}_{0}=x_{0}\|s\|, \\
& \dot{\hat{b}}_{1}=x_{1}\|s\|\|q\|, \\
& \dot{\widehat{b}}_{2}=x_{2}\|s\|\|\dot{q}\| .
\end{aligned}
$$

Substituting the adaptation laws (44) into (43) yields

$$
\begin{aligned}
\dot{V}= & s^{T} f-\|s\|\left(\widehat{b}_{0}+\widehat{b}_{1}\|q\|+\widehat{b}_{2}\|\dot{q}\|\right) \\
& -\left(\widetilde{b}_{0}\|s\|+\widetilde{b}_{1}\|s\|\|q\|+\widetilde{b}_{2}\|s\|\|\dot{q}\|\right) \\
= & s^{T} f-\|s\|\left(b_{0}+b_{1}\|q\|+b_{2}\|\dot{q}\|\right) \\
\leq & \|s\|\|f\|-\|s\|\left(b_{0}+b_{1}\|q\|+b_{2}\|\dot{q}\|\right) \\
= & \|s\|\left(\|f\|-\left(b_{0}+b_{1}\|q\|+b_{2}\|\dot{q}\|\right)\right) \\
\leq & 0,
\end{aligned}
$$

where $\dot{V}$ is the negative semidefinite, which implies that the Lyapunov function decreases gradually and the fractional fast TSM surface converges to zero in finite time. Namely, $V, s, \widetilde{D}, \widetilde{K}, \widetilde{\Omega}, \widetilde{b}_{i}$ are all bounded.

Remark 4. Assume that the upper bound of the lumped uncertainties $b_{0}+b_{1}\|q\|+b_{2}\|\dot{q}\|=\|f\|+\varepsilon$; then $\dot{V}_{1} \leq-\varepsilon\|s\|$. Integrating $\dot{V}$ with respect to time, we have $\int_{0}^{t}\|s\| \mathrm{d} t \leq 1 / \varepsilon[V(0)-V(t)]$. Since $V(0)$ is bounded and $V(t)$ is both bounded and nonincreasing, it is concluded that $\int_{0}^{t}\|s\| \mathrm{d} t$ is bounded. According to Barbalat's lemma, $\lim _{t \rightarrow \infty} s(t)=0$ and $\lim _{t \rightarrow \infty} e(t)=0$ can be achieved, which means the tracking error and fractional fast TSM surface will converge to zero in finite time.

Remark 5. In the adaptive control system design, the persistent excitation (PE) condition is an important factor to estimate parameters correctly. The reference trajectory that the gyroscope must follow is generated such that the 
resonance frequency of the $x$-axis is different from that of the $y$-axis, which satisfies the PE condition. If reference signals are persistently excited, then adaptive laws (44) guarantee that $\widetilde{D} \longrightarrow 0, \widetilde{K} \longrightarrow 0, \widetilde{\Omega} \longrightarrow 0$, which means $D, K, \Omega$ will converge to their true values. Then the unknown angular velocity as well as all other unknown parameters can also reach their actual values.

Remark 6. Similarly, in actual controller design, $\Delta u_{2}$ is designed as

$$
\Delta u_{2}= \begin{cases}-\frac{s}{\|s\|}\left(\widehat{b}_{0}+\widehat{b}_{1}\|q\|+\widehat{b}_{2}\|\dot{q}\|\right), & \|s\| \geq \delta, \\ -\frac{s}{\delta^{2}}\left(\|s\|\left(\widehat{b}_{0}+\widehat{b}_{1}\|q\|+\widehat{b}_{2}\|\dot{q}\|\right)\right), & \|s\|<\delta,\end{cases}
$$

where $\delta$ is a positive constant. A small boundary layer around the sliding surface will be created by this modification and then the chattering can be eliminated.

\section{Simulation Study}

In this section, the efficiency of the proposed robust adaptive fractional fast TSM control method is evaluated on a $z$-axis microgyroscope using Matlab/Simulink. Parameters of the microgyroscope are selected as follows:

$$
\begin{aligned}
m & =1.8 \times 10^{-7} \mathrm{~kg}, \\
d_{x x} & =1.8 \times 10^{-6} \mathrm{Ns} / \mathrm{m}, \\
d_{y y} & =1.8 \times 10^{-6} \mathrm{Ns} / \mathrm{m}, \\
d_{x y} & =3.6 \times 10^{-7} \mathrm{Ns} / \mathrm{m}, \\
k_{x x} & =63.955 \mathrm{~N} / \mathrm{m}, \\
k_{y y} & =95.92 \mathrm{~N} / \mathrm{m}, \\
k_{x y} & =12.779 \mathrm{~N} / \mathrm{m} .
\end{aligned}
$$

Suppose that the reference length $q_{0}$ is $1 \mu \mathrm{m}, \omega_{0}$ is $1 \mathrm{kHZ}$, and $\Omega_{z}$ is $100 \mathrm{rad} / \mathrm{s}$; then, the nondimensional gyroscope parameter matrices can be derived as follows:

$$
\begin{aligned}
D & =\left[\begin{array}{cc}
0.01 & 0.002 \\
0.002 & 0.01
\end{array}\right], \\
K & =\left[\begin{array}{ll}
355.3 & 70.99 \\
70.99 & 532.9
\end{array}\right], \\
\Omega & =\left[\begin{array}{cc}
0 & -0.1 \\
0.1 & 0
\end{array}\right] .
\end{aligned}
$$

The objective of this controller is to track the desired motion trajectories $q_{r 1}=\sin (\pi t), q_{r 2}=\cos (0.5 \pi t)$ with the initial states of the system:

$$
\begin{aligned}
& q_{1}(0)=0.5, \\
& \dot{q}_{1}(0)=0, \\
& q_{2}(0)=0.5, \\
& \dot{q}_{2}(0)=0 .
\end{aligned}
$$

The initial conditions of $D, K, \Omega$ matrices are

$$
\begin{aligned}
& D_{0}=0.95 * D, \\
& K_{0}=0.95 * K, \\
& \Omega_{0}=0.95 * \Omega .
\end{aligned}
$$

Select the fractional fast TSM surface parameters as

$$
\begin{aligned}
\lambda_{1} & =10, \\
\lambda_{2} & =0.5, \\
\gamma & =0.5 .
\end{aligned}
$$

The initial conditions of the upper bound of the lumped uncertainties are

$$
\begin{aligned}
& b_{00}=0.1, \\
& b_{10}=0.2, \\
& b_{20}=0.3 .
\end{aligned}
$$

The adaptive gains are

$$
\begin{aligned}
& M=N=P=\operatorname{diag}(150,150), \\
& x_{0}=1, \\
& x_{1}=1.6, \\
& x_{2}=1.6 .
\end{aligned}
$$

The boundary layer $\delta$ is designed as 0.06 so as to alleviate the chattering phenomenon. When the fractional-order $\alpha$ is set as 0.9 and the lumped uncertainties are applied as random signal $f=[\operatorname{randn}(1,1)$; $\operatorname{randn}(1,1)]$, the corresponding simulation is conducted to demonstrate the performance of the proposed adaptive fractional fast TSMC and compare it with existing conventional fast TSMC and fractional SMC [52]. Simulation results are plotted as Figures 3-16.

The plots of position tracking trajectory are shown in Figure 3. It is intuitive to see that the states of the system track the desired trajectories completely within a short time. Figures 4-6 show the tracking errors under the robust adaptive fractional fast TSM control scheme, the adaptive fractional sliding mode control, and the conventional fast TSM control scheme, respectively. It is observed that fractional fast TSMC makes tracking error converge to zero in less than 5 seconds, while fractional SMC needs about 10 seconds to do that, which means fractional fast TSMC obtains faster convergence. Meanwhile it can follow desired trajectories with higher accuracy compared to integer-order fast TSMC by comparing the tracking error.

The corresponding fractional fast TSM surface is illustrated in Figure 7. It is obvious that the designed sliding surface converges to zero in finite time, which confirms the fast convergence of the system and guarantees that the states of the system attain to the sliding surface. In contrast, the convergence of conventional fast TSM surface drawn in Figure 8 is not as smooth as fractional fast TSM surface. Figure 9 plots the control input signals of the microgyroscope without any chattering using a boundary layer. In Figures 10 and 13, the estimations of the system parameter 


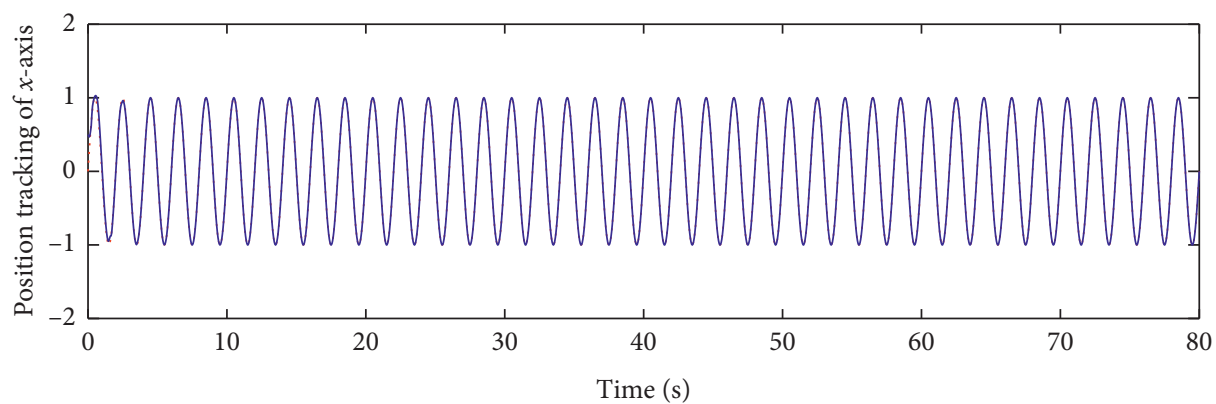

…. $q r_{1}(t)$

- $q_{1}(t)$

(a)

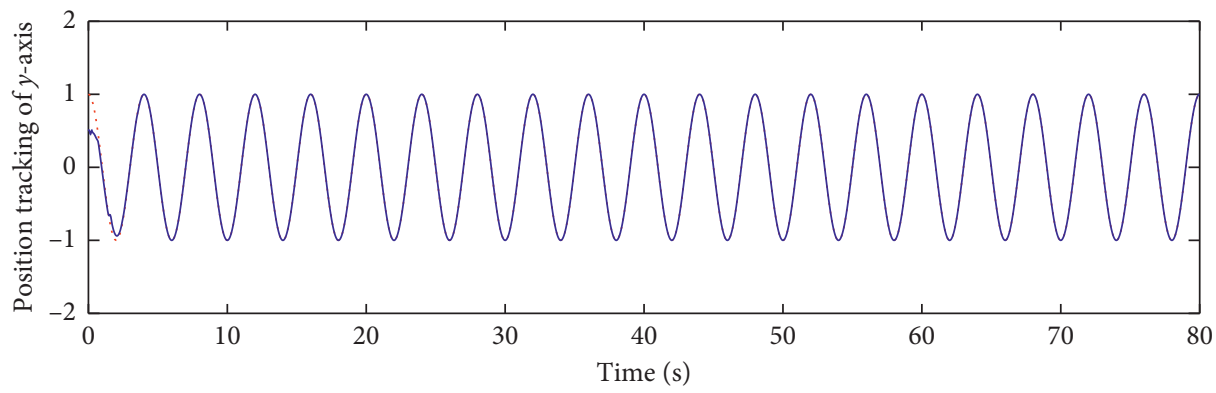

…. $q r_{2}(t)$

$-q_{2}(t)$

(b)

FIGURE 3: Tracking trajectory using fractional fast TSM surface.

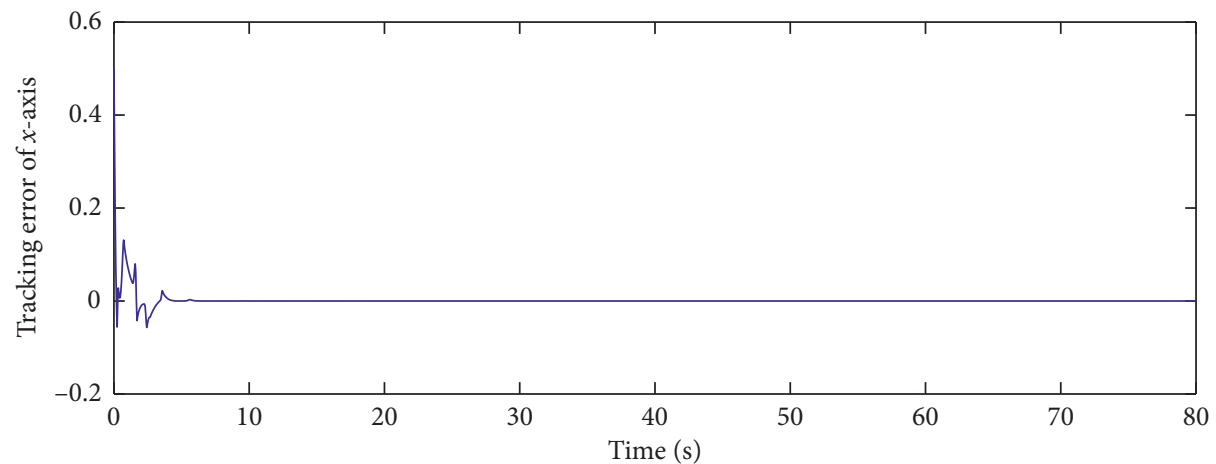

(a)

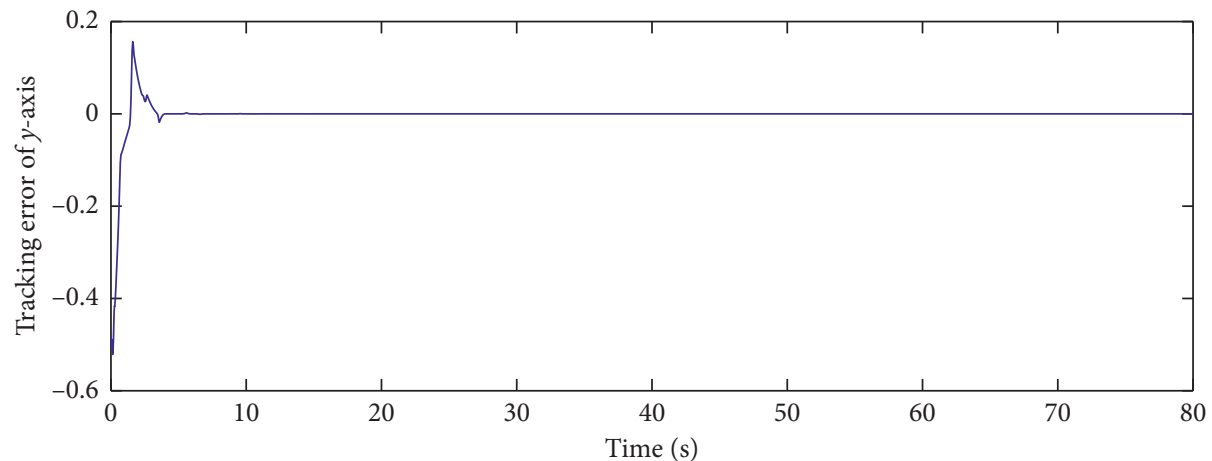

(b)

FIgURE 4: Tracking error using fractional fast TSM surface. 


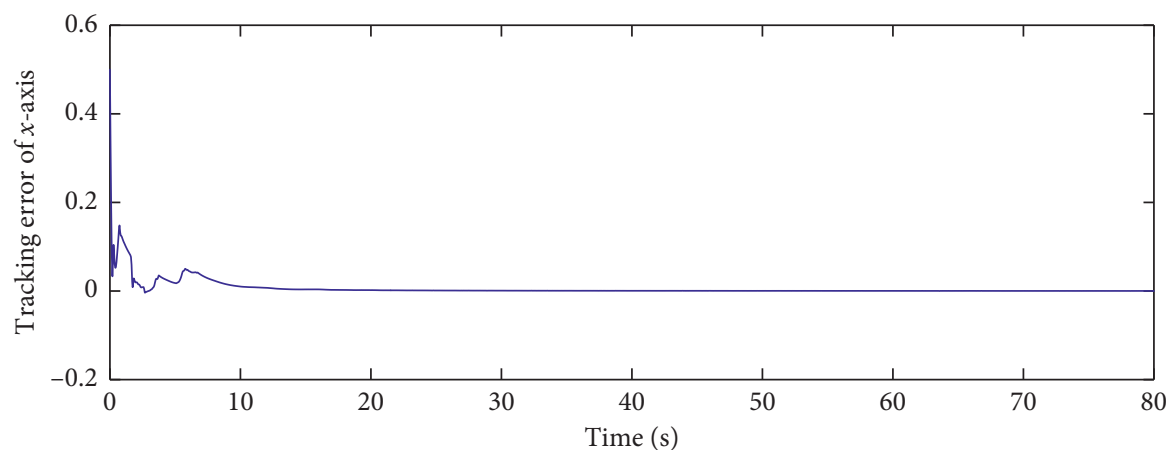

(a)

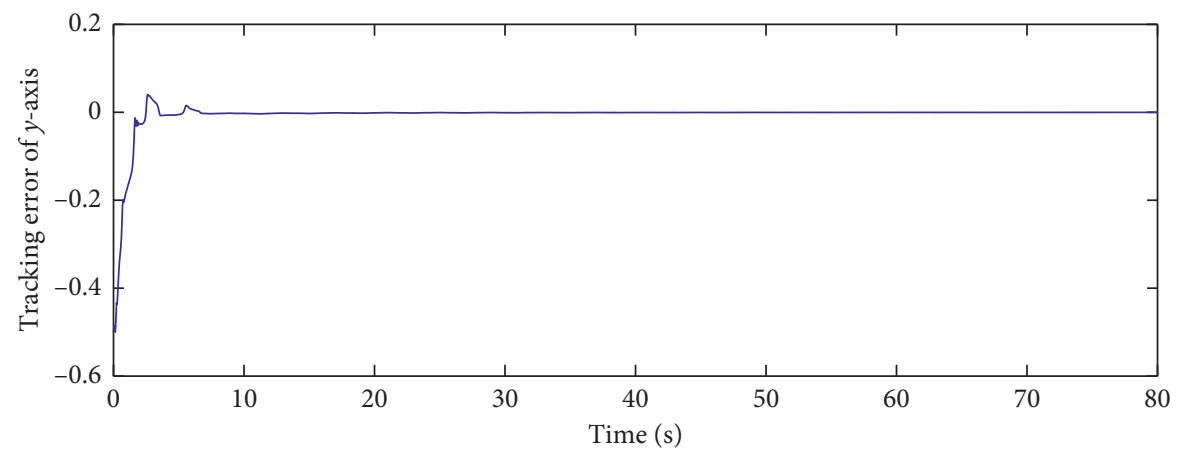

(b)

FIGURE 5: Tracking error using fractional sliding surface.

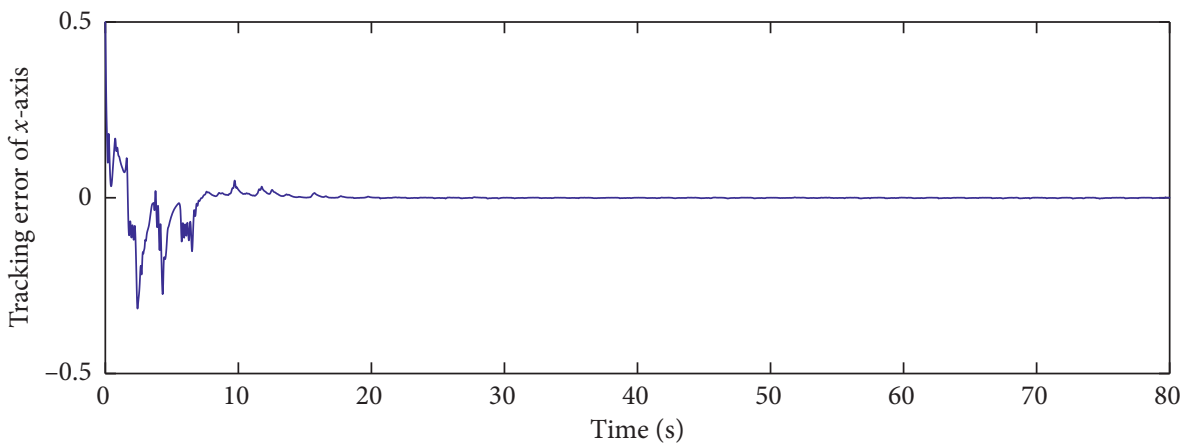

(a)

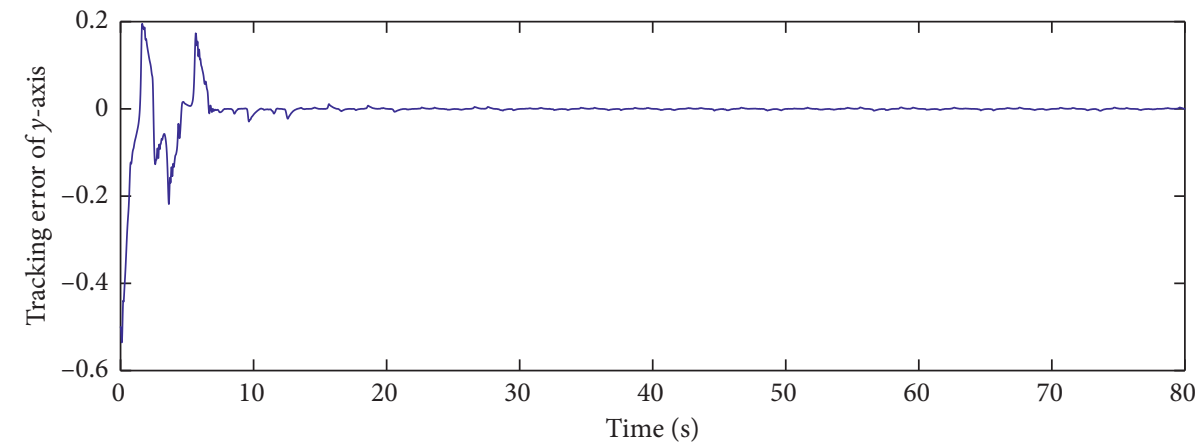

(b)

FIGURE 6: Tracking error using conventional fast TSM surface. 


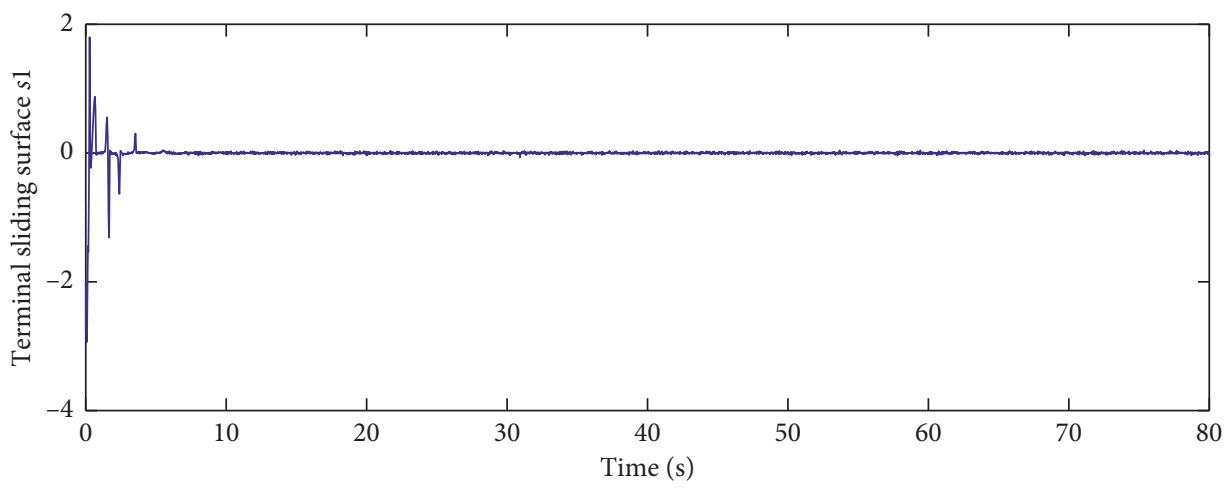

(a)

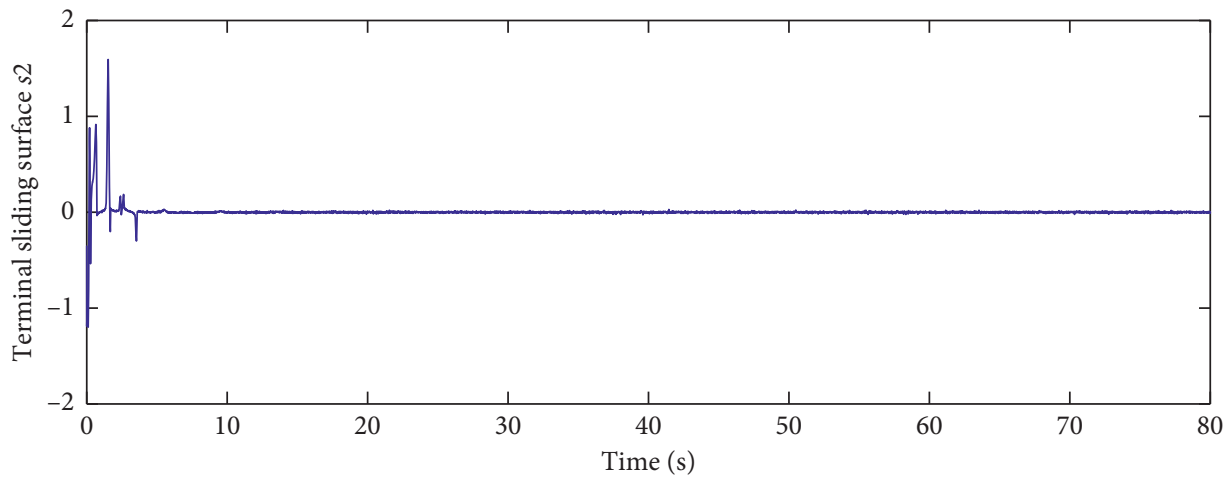

(b)

FIgURE 7: Fractional fast TSM surface.

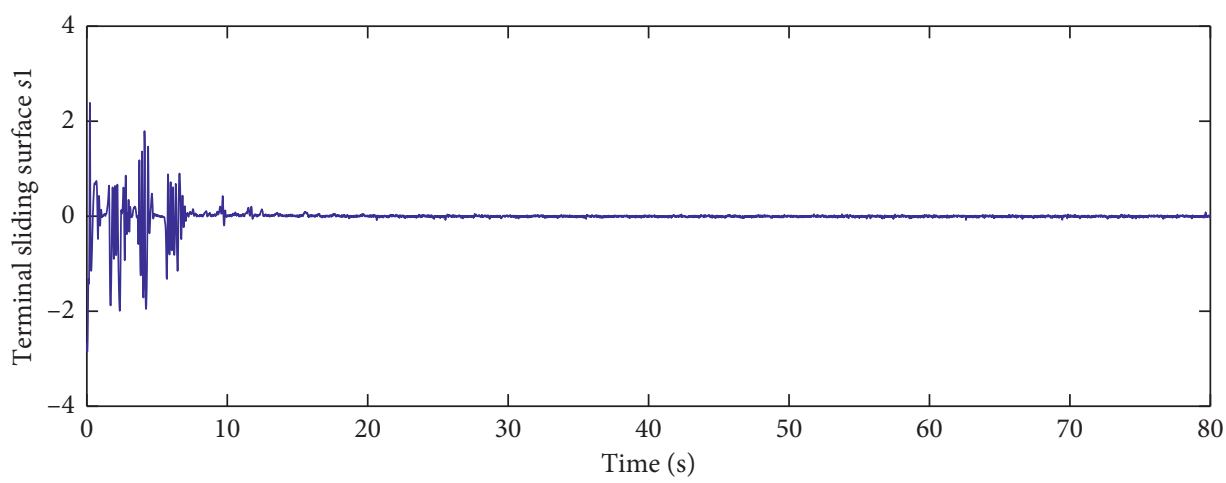

(a)

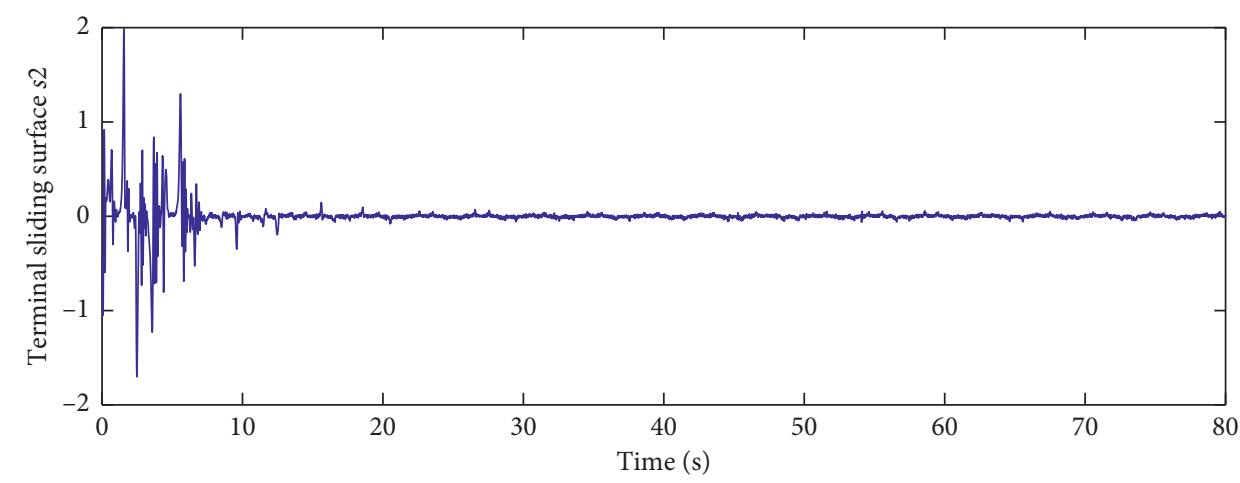

(b)

FIgUre 8: Conventional fast TSM surface. 


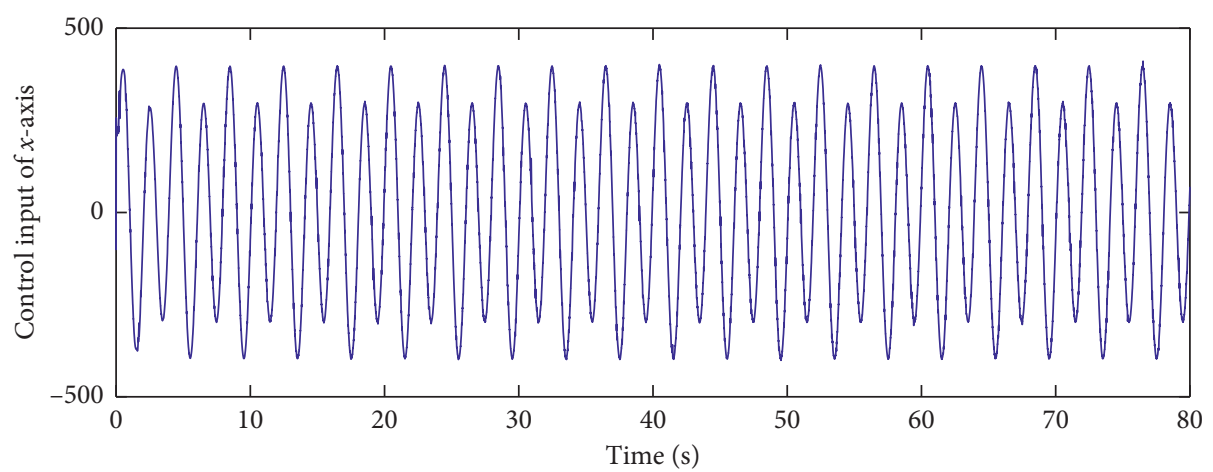

(a)

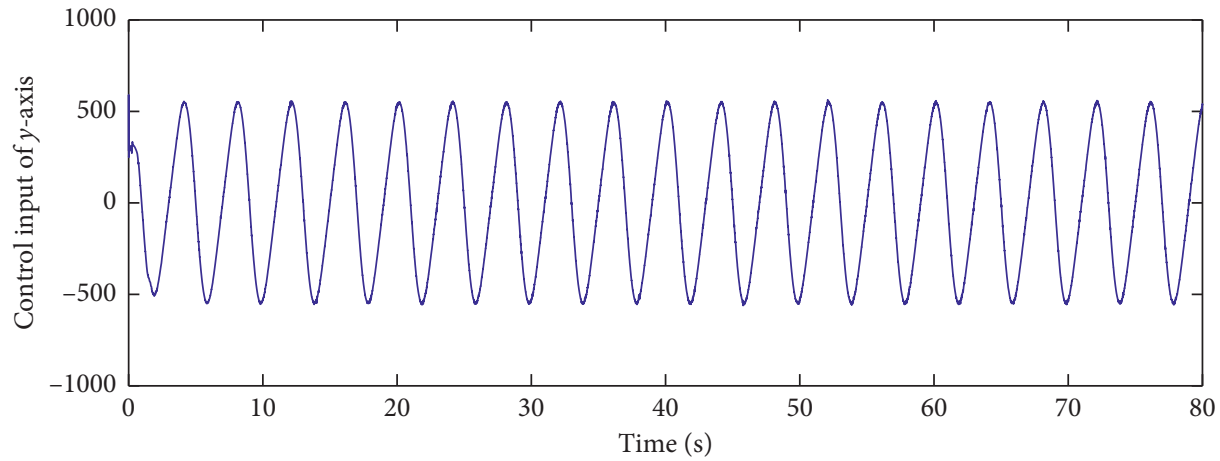

(b)

Figure 9: Control input signals using fractional fast TSMC.

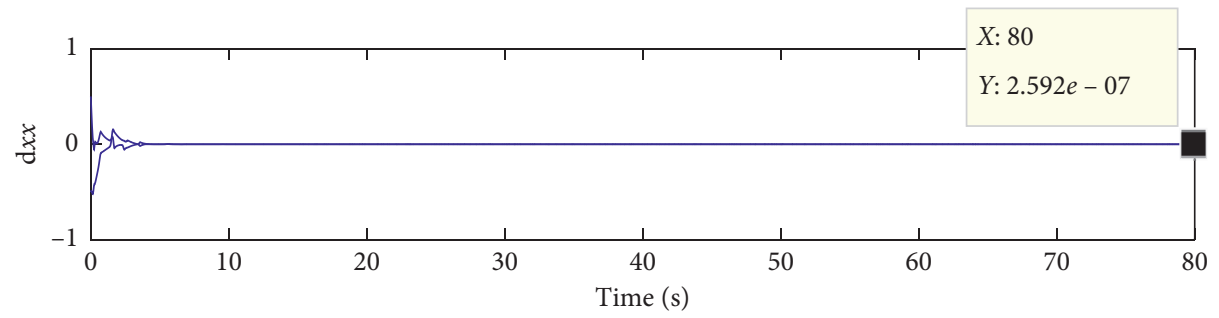

(a)

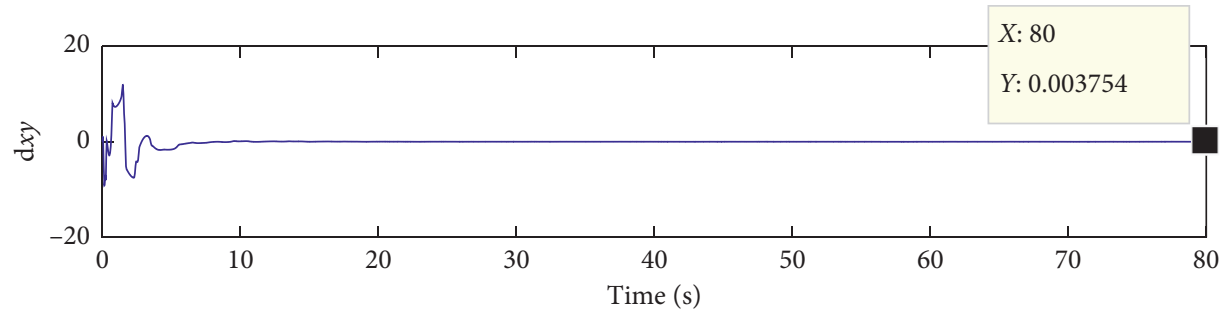

(b)

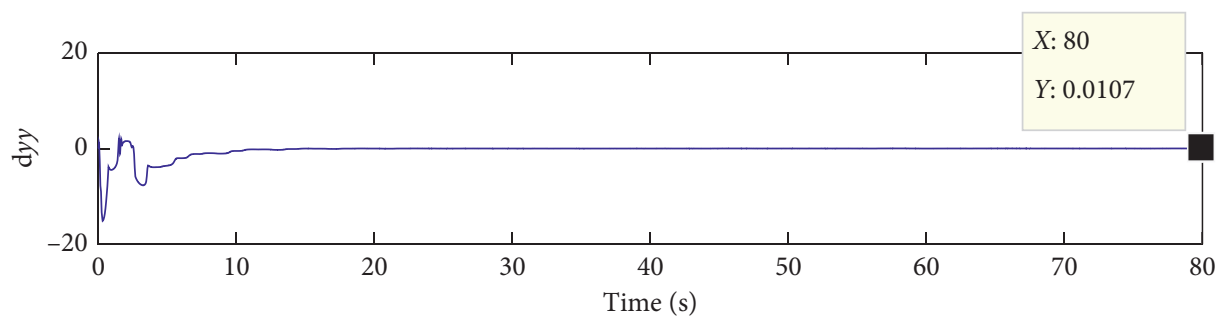

(c)

Figure 10: Adaptation of damping coefficients using fractional fast TSMC. 


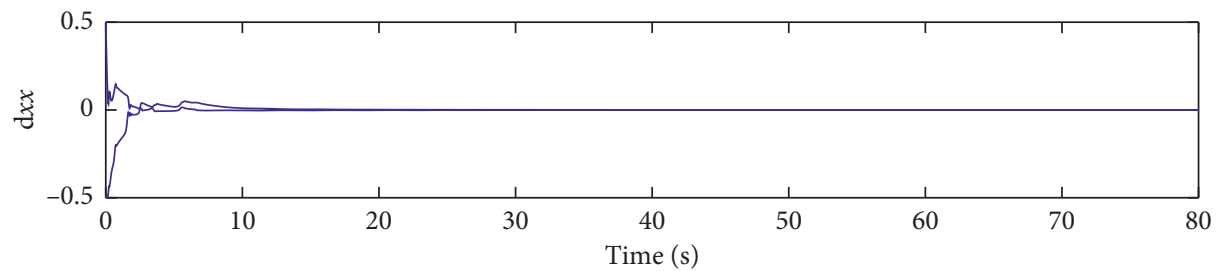

(a)

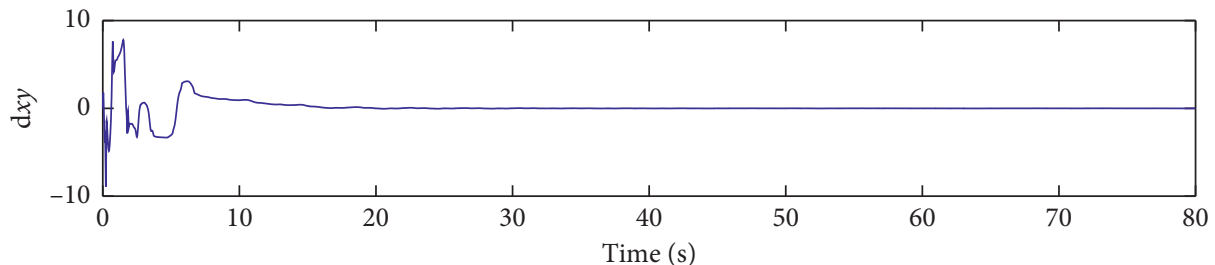

(b)

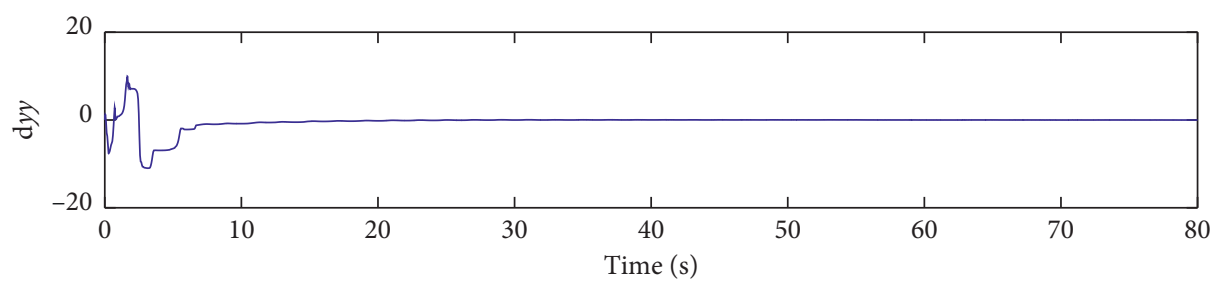

(c)

FIgURE 11: Adaptation of damping coefficients using fractional SMC.

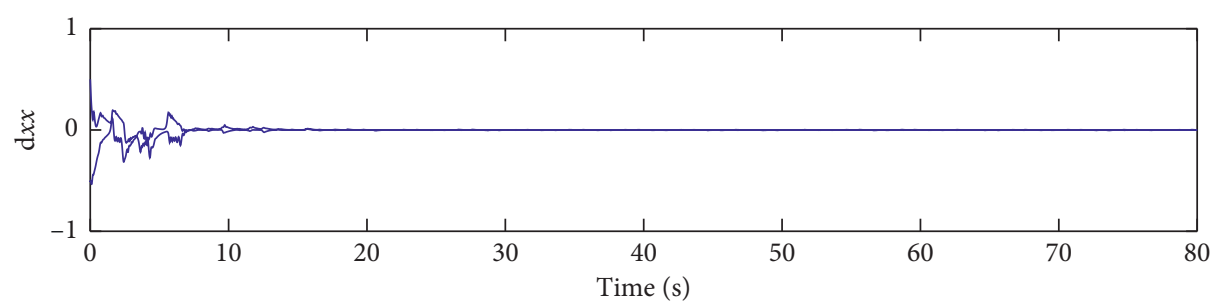

(a)

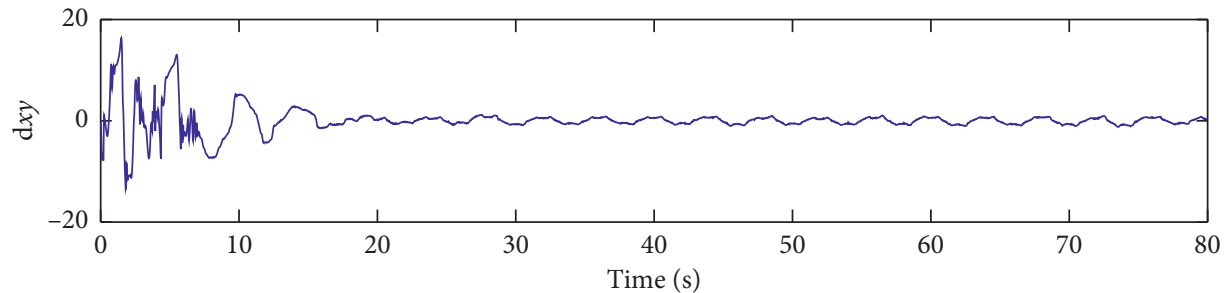

(b)

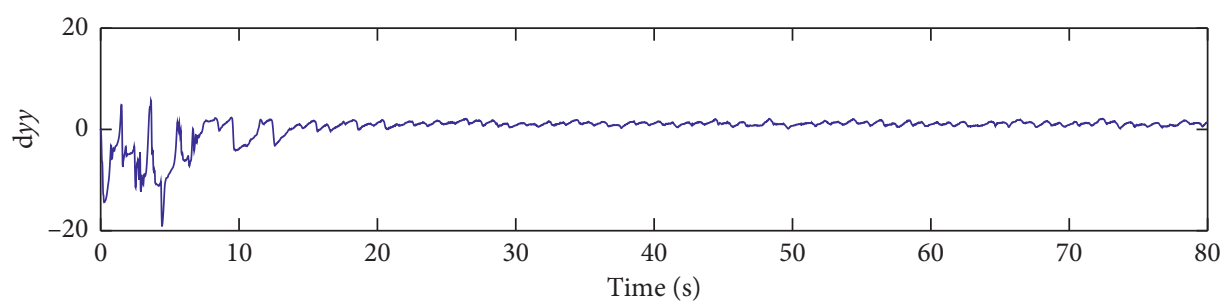

(c)

FIgURE 12: Adaptation of damping coefficients using conventional fast TSMC. 


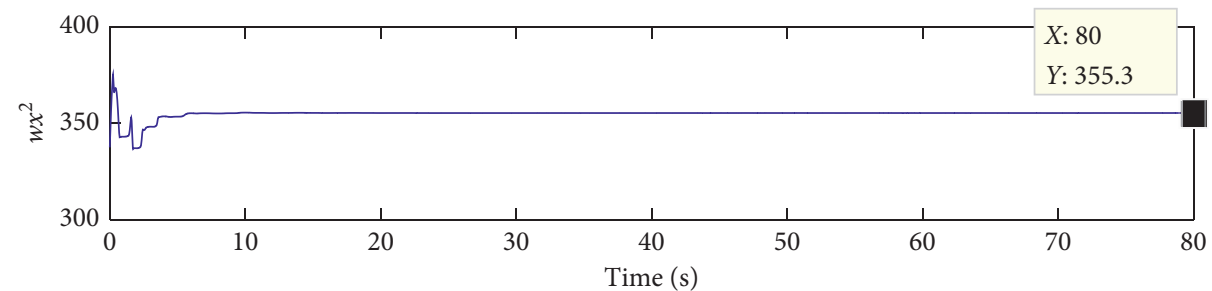

(a)

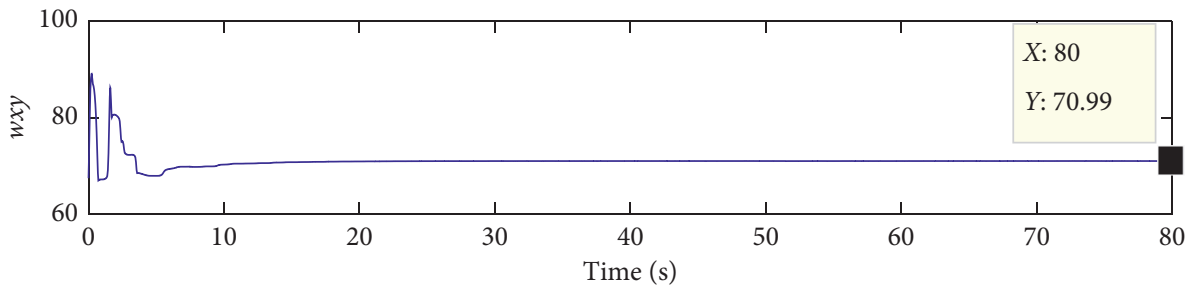

(b)

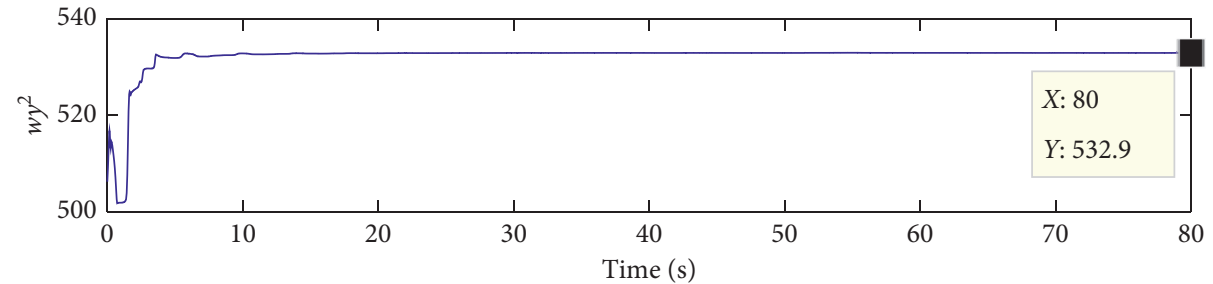

(c)

Figure 13: Adaptation of spring constants using fractional fast TSMC.

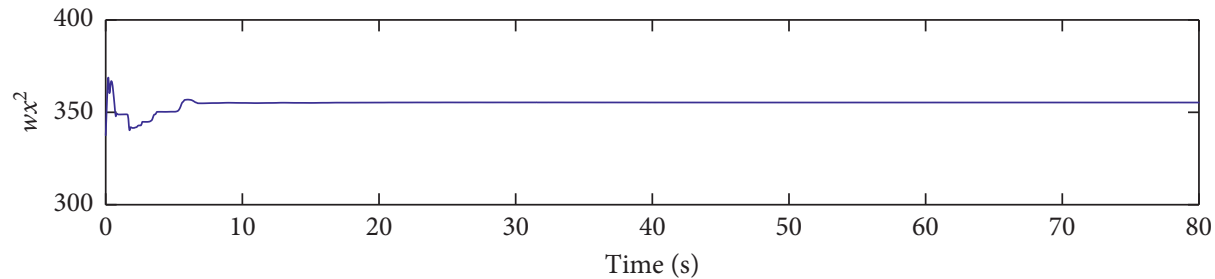

(a)

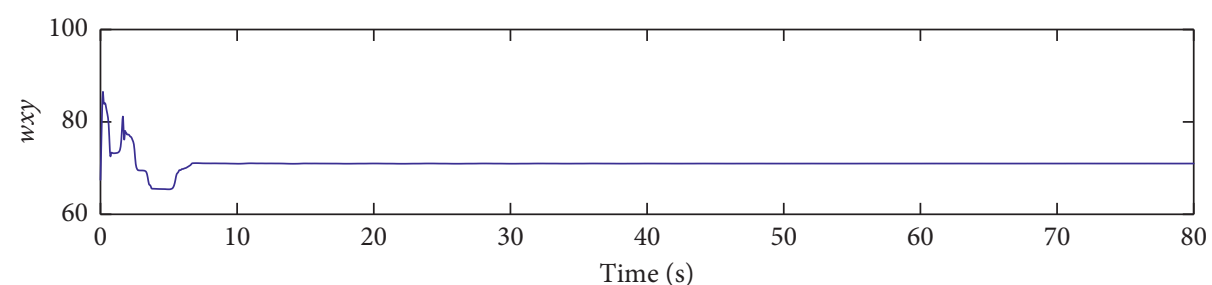

(b)

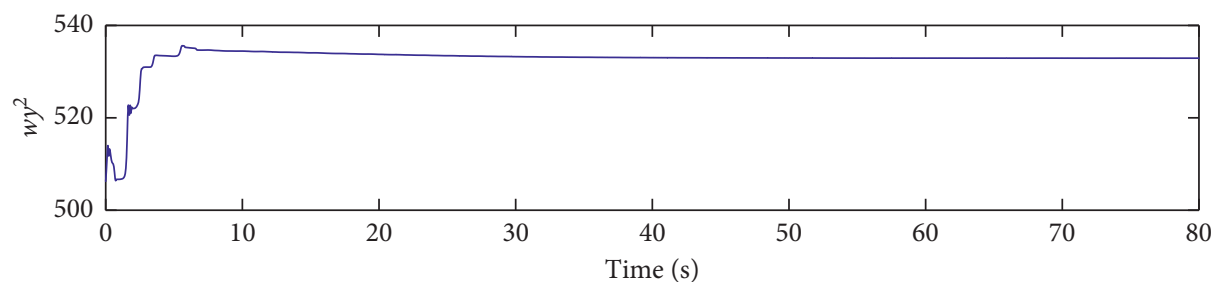

(c)

Figure 14: Adaptation of spring constants using fractional SMC. 


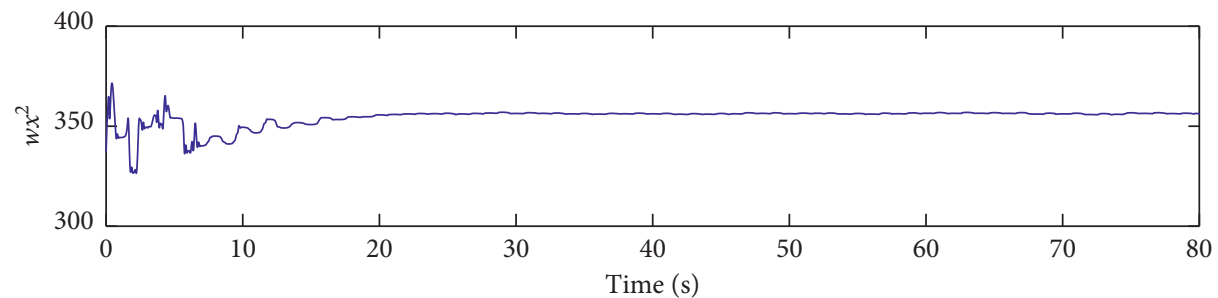

(a)

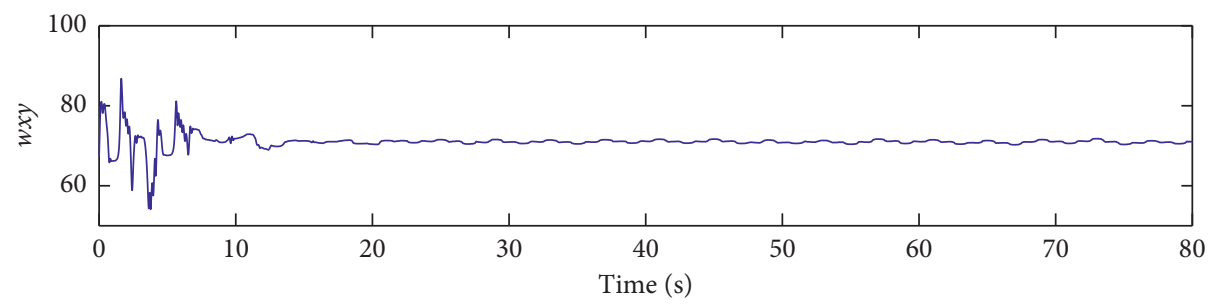

(b)

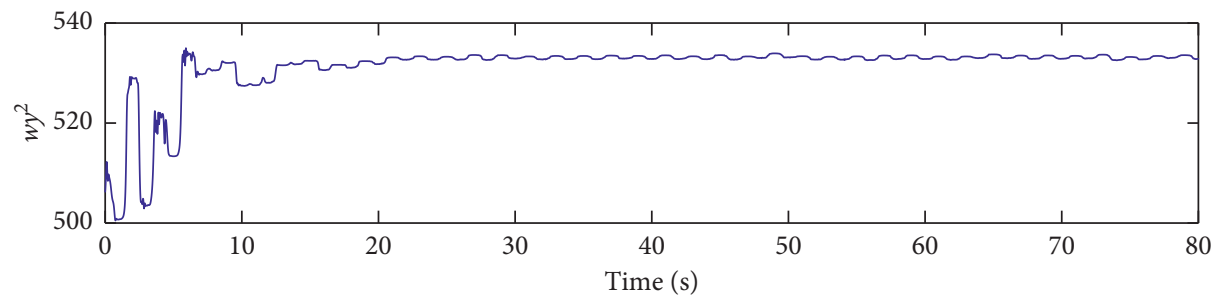

(c)

FIgURE 15: Adaptation of spring constants using conventional fast TSMC.

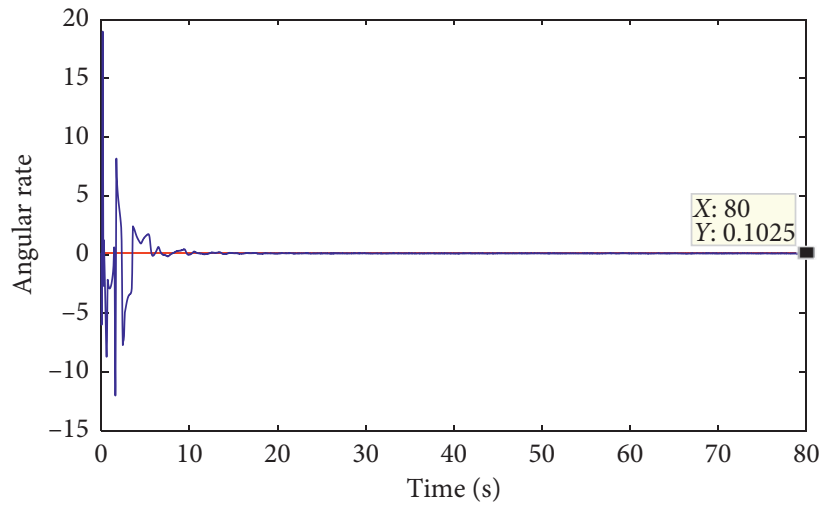

FIgURe 16: Adaptation of angular velocity using fractional fast TSMC.

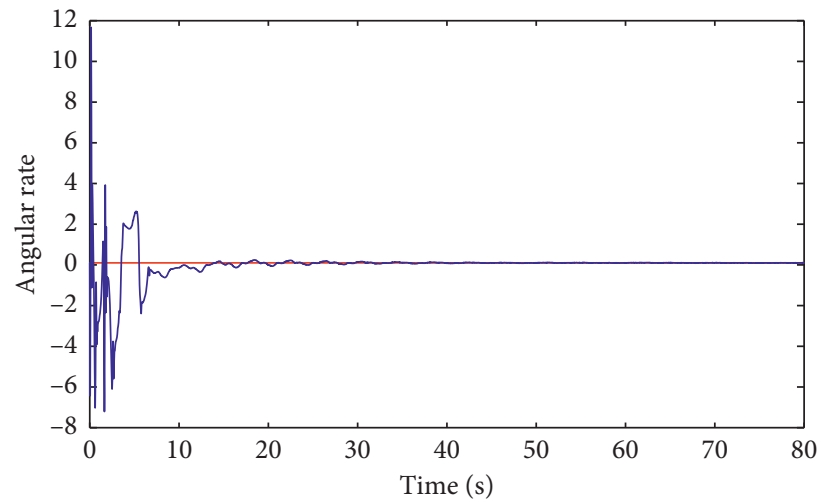

Figure 17: Adaptation of angular velocity using fractional SMC. 


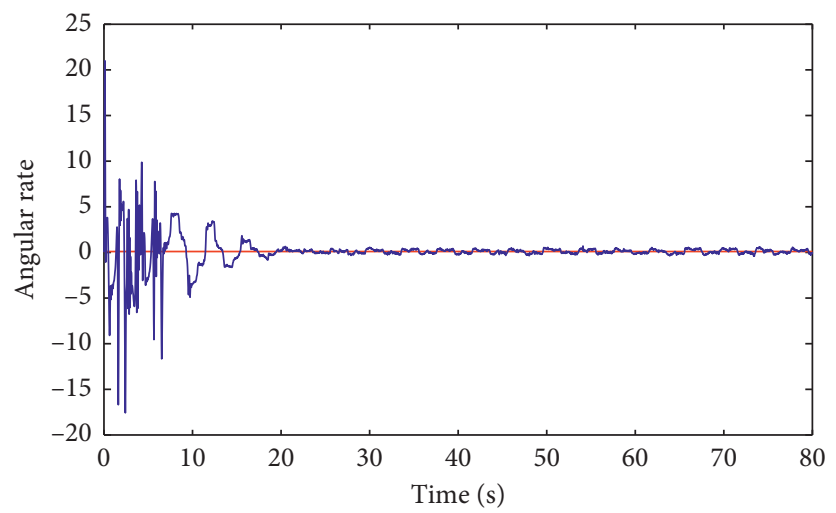

FIgURE 18: Adaptation of angular velocity using conventional fast TSCM.

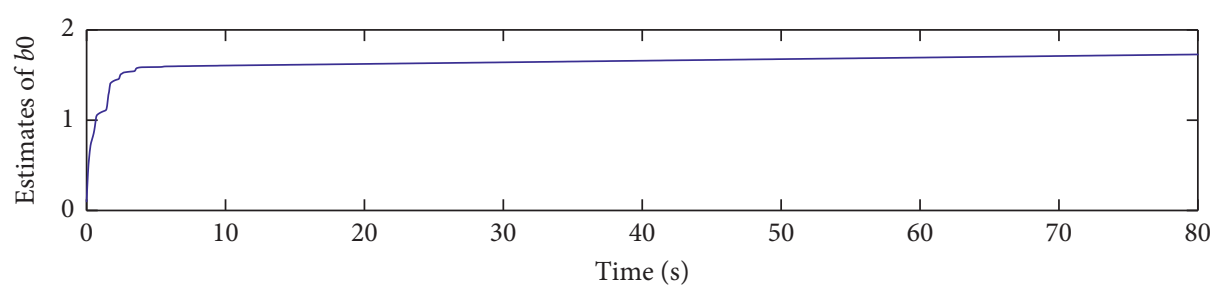

(a)

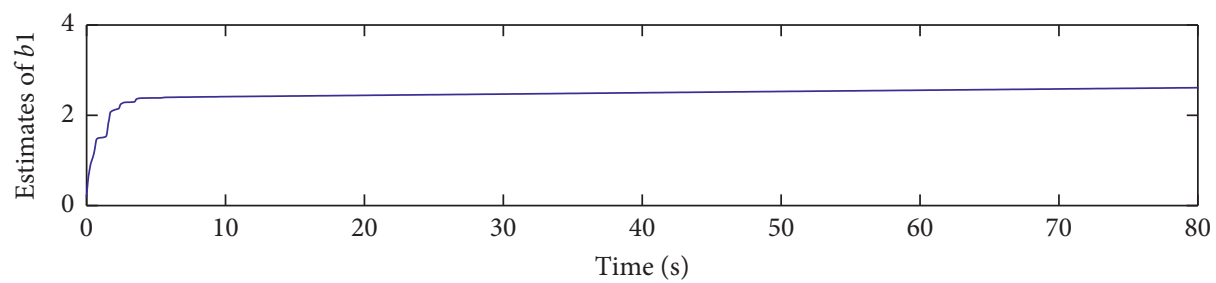

(b)

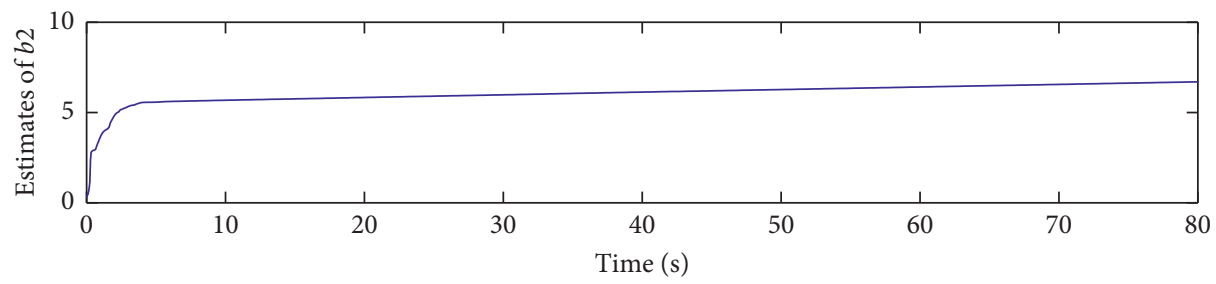

(c)

Figure 19: Adaptively estimated parameters $\widehat{b}_{0}, \widehat{b}_{1}, \widehat{b}_{2}$.

matrices $D$ and $K$ using fractional fast TSMC are depicted, respectively, which have been verified to converge to their true values in a shorter time compared to fractional SMC as seen in Figures 11 and 14. In addition, Figures 12 and 15 show the estimate of spring and damping coefficients under conventional fast TSMC whose estimation accuracy is relatively lower than that of fractional fast TSMC and fractional SMC. Figures 16-18 compare the adaptation of angular velocity among fractional fast TSMC, fractional SMC, and conventional fast TSMC whose estimates converge to the true values fleetly. However, it is observed that the estimate of angular velocity using fractional fast TSMC has better convergence performance in comparison with fractional SCM and conventional fast TSMC. Figure 19 describes the adaptation of the upper bound parameters $\widehat{b}_{0}, \widehat{b}_{1}, \widehat{b}_{2}$, which also shows stable convergence. According to the comparison above, it is obvious that fractional calculus incorporated into control method can improve the accuracy and performance of the system. Meanwhile, convergence time of the fast terminal sliding mode control is shorter than that of traditional sliding mode control.

A universal standard is adopted to quantify tracking error by calculating root mean square error (RMSE). The RMSE reflects how much the measured value deviates from its true value and the smaller the RMSE is, the higher the measurement accuracy is. For intuitively comparing the tracking performance under adaptive integer-order fast TSMC and adaptive fractional fast TSM controller, we added 
TABLE 1: RMSE of $x$-axis and $y$-axis under different fractional orders and integer orders.

\begin{tabular}{lcc}
\hline RMSE $\alpha$ & $X$ & $Y$ \\
\hline 0.1 & 0.0281 & 0.0386 \\
0.3 & 0.0277 & 0.0391 \\
0.5 & 0.0298 & 0.0415 \\
0.7 & 0.0251 & 0.0386 \\
0.9 & 0.0220 & 0.0384 \\
1.0 & 0.0306 & 0.0462 \\
\hline
\end{tabular}

data comparison by calculating the RMSE under these two methods. Table 1 describes the RMSE along $x$-axis and $y$-axis under different orders, where $\alpha=1$ means it is an adaptive integer-order fast TSMC, and when $\alpha$ is a fraction it is an adaptive fractional fast TSM controller.

It is clear from Table 1 that the trajectories of adaptive fractional fast TSM controller are closer to the reference signal than the integer-order fast TSMC. Meanwhile, better tracking performance can be achieved by adjusting the fractional-order $\alpha$. The RMSE seems to be minimal in the case of $\alpha=0.9$; that is the reason why we select $\alpha=0.9$ in the previous controller design. However, the computational load of the fractional order is larger than integerorder ones.

\section{Conclusion}

In this paper, a novel fractional intelligent control strategy comprised of adaptive control and fast terminal sliding mode control is designed to achieve the fast response performance with robustness against uncertainties for trajectory tracking of microgyroscope in the presence of uncertainties and external disturbances. A fractional differential fast terminal sliding surface is defined, which ensures finite convergence and higher control accuracy compared to conventional integer-order ones. For achieving no chattering, a boundary layer around the switching manifold is added and continuous control laws are utilized inside the boundary. In addition, adaptive schemes are used to estimate the unknown system parameters and the upper bound uncertainties. Simulations under different fractional orders are performed to find the best fractional order for the better performance, and comparison with the other exiting methods demonstrates the superiorities of the proposed algorithm. The next step is to implement and verify the proposed robust adaptive fractional fast terminal sliding mode controller on a realtime hardware experiment.

\section{Data Availability}

All relevant data are included within the paper.

\section{Conflicts of Interest}

The authors declare that there are no conflicts of interest regarding the publication of this paper.

\section{Acknowledgments}

This work was supported by the National Science Foundation of China under Grant no. 61873085.

\section{References}

[1] K. Ma and M. N. Ghasemi-Nejhad, "Adaptive control of flexible active composite manipulators driven by piezoelectric patches and active struts with dead zones," IEEE Transactions on Control Systems Technology, vol. 16, no. 5, pp. 897-907, 2008.

[2] R. M. Sanner and J. J.-E. Slotine, "Gaussian networks for direct adaptive control," in Proceedings of the 2009 American Control Conference, pp. 2153-2159, Boston, MA, USA, June 1991.

[3] Y.-J. Liu, M. Gong, S. Tong, C. L. P. Chen, and D.-J. Li, "Adaptive fuzzy output feedback control for a class of nonlinear systems with full state constraints," IEEE Transactions on Fuzzy Systems, vol. 26, no. 5, pp. 2607-2617, 2018.

[4] X. Hu, J. Du, and Y. Sun, "Robust adaptive control for dynamic positioning of ships," IEEE Journal of Oceanic Engineering, vol. 42, no. 4, pp. 826-835, 2017.

[5] F. Tu, S. Sam Ge, Y. S. Choo, and C. C. Hang, "Adaptive dynamic positioning control for accommodation vessels with multiple constraints," IET Control Theory \& Applications, vol. 11, no. 3, pp. 329-340, 2017.

[6] G. Zhang, Y. Deng, W. Zhang, and C. Huang, "Novel DVS guidance and path-following control for underactuated ships in presence of multiple static and moving obstacles," Ocean Engineering, vol. 170, no. 11, pp. 100-110, 2018.

[7] S. Ahmed, H. Wang, M. S. Aslam, I. Ghous, and I. Qaisar, "Robust adaptive control of robotic manipulator with input time-varying delay," International Journal of Control, Automation and Systems, vol. 17, no. 9, pp. 2193-2202, 2019.

[8] H. Wang, "Adaptive control of robot manipulators with uncertain kinematics and dynamics," IEEE Transactions on Automatic Control, vol. 62, no. 2, pp. 948-954, 2017.

[9] J. Yu, B. Chen, H. Yu, C. Lin, Z. Ji, and X. Cheng, "Position tracking control for chaotic permanent magnet synchronous motors via indirect adaptive neural approximation," Neurocomputing, vol. 156, pp. 245-251, 2015.

[10] Y. Fang, J. Fei, and T. Hu, "Adaptive backstepping fuzzy sliding mode vibration control of flexible structure," Journal of Low Frequency Noise, Vibration and Active Control, vol. 37, no. 4, pp. 1079-1096, 2018.

[11] H.-N. Wu and Z.-P. Wang, "Observer-based," IEEE Transactions on Fuzzy Systems, vol. 26, no. 2, pp. 454-473, 2018.

[12] Y. Li and S. Tong, "Adaptive neural networks prescribed performance control design for switched interconnected uncertain nonlinear systems," IEEE Transactions on Neural Networks and Learning Systems, vol. 29, no. 7, pp. 3059-3068, 2017.

[13] H. Wang, P. Liu, and B. Niu, "Robust fuzzy adaptive tracking control for nonaffine stochastic nonlinear switching systems," IEEE Transactions on Cybernetics, vol. 48, no. 8, pp. 24622471, 2018.

[14] J. Fei and Z. Feng, "Adaptive fuzzy super-twisting sliding mode control for microgyroscope," Complexity, vol. 2019, Article ID 6942642, 13 pages, 2019.

[15] J. Fei and W. Yan, "Adaptive control of MEMS gyroscope using global fast terminal sliding mode control and fuzzyneural-network," Nonlinear Dynamics, vol. 78, no. 1, pp. 103116, 2014. 
[16] Y. Fang, J. Fei, and Y. Yang, "Adaptive backstepping design of a microgyroscope," Micromachines, vol. 9, no. 7, pp. 338-345, 2018.

[17] L. Wu, S. Mazumder, and O. Kaynak, "Sliding mode control and observation for complex industrial systems-part II," IEEE Transactions on Industrial Electronics, vol. 65, no. 1, pp. 830-833, 2018.

[18] J. Fei and H. Ding, "Adaptive sliding mode control of dynamic system using RBF neural network," Nonlinear Dynamics, vol. 70, no. 2, pp. 1563-1573, 2012.

[19] Y. Han, Y. Kao, and C. Gao, "Robust sliding mode control for uncertain discrete singular systems with time-varying delays and external disturbances," Automatica, vol. 75, no. 4, pp. 210-216, 2017.

[20] B. Lu, Y. Fang, and N. Sun, "Continuous sliding mode control strategy for a class of nonlinear underactuated systems," IEEE Transactions on Automatic Control, vol. 63, no. 10, pp. 3471-3478, 2018.

[21] J. Fei and Y. Chu, "Double hidden layer recurrent neural adaptive global sliding mode control of active power filter," IEEE Transactions on Power Electronics, vol. 35, no. 3, pp. 3069-3084, 2020.

[22] J. Fei and Y. Chen, "Dynamic terminal sliding mode control for single-phase Active power filter using double hidden layer recurrent neural network," IEEE Transactions on Power Electronics, vol. 35, no. 9, pp. 9906-9924, 2020.

[23] J. Fei and H. Wang, "Experimental investigation of recurrent neural network fractional-order sliding mode control for active power filter," IEEE Transactions on Circuits and Systems II-Express Briefs, 2019.

[24] Y. Chu, J. Fei, and S. Hou, "Adaptive global sliding mode control for dynamic systems using double hidden layer recurrent neural network structure," IEEE Transactions on Neural Network and Learning System, vol. 31, no. 4, pp. 1297-1309, 2020.

[25] J. Fei and Z. Feng, "Fractional-order finite-time supertwisting sliding mode control of micro gyroscope based on double loop fuzzy neural network," IEEE Transactions on Systems, Man, and Cybernetics: Systems, 2020.

[26] Y. Zhu and J. Fei, "Disturbance observer based fuzzy sliding mode control of PV grid connected inverter," IEEE Access, vol. 6, pp. 21202-21211, 2018.

[27] C. Yang, "Robust adaptive terminal sliding mode synchronized control for a class of non-autonomous chaotic systems," Asian Journal of Control, vol. 15, no. 6, pp. 1677-1685, 2013.

[28] M. B. R. Neila and D. Tarak, "Adaptive terminal sliding mode control for rigid robotic manipulators," International Journal of Automation and Computing, vol. 8, no. 2, pp. 215-220, 2011.

[29] V. Nekoukar and A. Erfanian, "Adaptive fuzzy terminal sliding mode control for a class of MIMO uncertain nonlinear systems," Fuzzy Sets \& Systems, vol. 179, no. 1, pp. 34-49, 2011.

[30] A. Saim, H. Wang, and Y. Tian, "Adaptive high-order terminal sliding mode control based on time delay estimation for the robotic manipulators with backlash hysteresis," IEEE Transactions on Systems Man \& Cybernetics Systems, pp. 1-10, 2019.

[31] L. Qiao and W. Zhang, "Trajectory tracking control of AUVs via adaptive fast nonsingular integral terminal sliding mode control," IEEE Transactions on Industrial Informatics, vol. 16, no. 2, pp. $1248-1258,2020$.

[32] L. Qiao and W. Zhang, "Double-loop integral terminal sliding mode tracking control for UUVs with adaptive dynamic compensation of uncertainties and disturbances," IEEE Journal of Oceanic Engineering, vol. 44, no. 1, pp. 29-53, 2019.

[33] L. Qiao and W. Zhang, "Adaptive second-order fast nonsingular terminal sliding mode tracking control for fully actuated autonomous underwater vehicles," IEEE Journal of Oceanic Engineering, vol. 44, no. 2, pp. 363-385, 2019.

[34] Y. Wang, K. Zhu, B. Chen, and M. Jin, "Model-free continuous nonsingular fast terminal sliding mode control for cable-driven manipulators," ISA Transactions, vol. 98, pp. 483-495, 2020.

[35] Y. Wang, S. Li, D. Wang, F. Ju, B. Chen, and H. Wu, “Adaptive time-delay control for cable-driven manipulators with enhanced nonsingular fast terminal sliding mode," IEEE Transactions on Industrial Electronics, p. 1, 2020.

[36] S. Li, M. Zhou, and X. Yu, "Design and implementation of terminal sliding mode control method for PMSM speed regulation system," IEEE Transactions on Industrial Informatics, vol. 9, no. 4, pp. 1879-1891, 2013.

[37] C. Solis, J. Clempner, and A. Poznyak, "Fast terminal sliding mode control with integral filter applied to a van der Pol oscillator," IEEE Transactions on Industrial Electronics, vol. 64, no. 7, pp. 5622-5628, 2017.

[38] J. Fei and X. Liang, "Adaptive backstepping fuzzy-neuralnetwork fractional order control of microgyroscope using nonsingular terminal sliding mode controller," Complexity, vol. 2018, Article ID 5246074, 12 pages, 2018.

[39] Y. Fang, J. Fei, and Di Cao, “Adaptive fuzzy-neural fractionalorder current control of active power filter with finite-time sliding," Controller, International Journal of Fuzzy System, vol. 21, no. 5, pp. 1533-1543, 2019.

[40] J. Fei and C. Lu, "Adaptive fractional order sliding mode controller with neural estimator," Journal of the Franklin Institute, vol. 355, no. 5, pp. 2369-2391, 2018.

[41] A. Saim, H. Wang, and Y. Tian, "Robust adaptive fractionalorder terminal sliding mode control for lower-limb exoskeleton," Asian Journal of Control, vol. 21, no. 1, pp. 473-482, 2018.

[42] L. Chen, R. Wu, and Y. He, "Adaptive sliding-mode control for fractional-order uncertain linear systems with nonlinear disturbances," Nonlinear Dynamics, vol. 80, no. 1-2, pp. 51-58, 2015.

[43] D. Nojavanzadeh and M. Badamchizadeh, "Adaptive fractional-order non-singular fast terminal sliding mode control for robot manipulators," IET Control Theory \& Applications, vol. 10, no. 13, pp. 1565-1572, 2016.

[44] G. Sun and Z. Ma, "Practical tracking control of linear motor with adaptive fractional order terminal sliding mode control," IEEE/ASME Transactions on Mechatronics, vol. 22, no. 6, pp. 2643-2653, 2017.

[45] M. Rahmani, "MEMS gyroscope control using a novel compound robust control," ISA Transactions, vol. 72, pp. 37-43, 2018.

[46] S. Ahmed, H. Wang, and Y. Tian, "Model-free control using time delay estimation and fractional-order nonsingular fast terminal sliding mode for uncertain lower-limb exoskeleton," Journal of Vibration and Control, vol. 24, no. 22, pp. 52735290, 2018.

[47] Y. Wang, L. Liu, D. Wang, and F. Ju, "Time-delay control using a novel nonlinear adaptive law for accurate trajectory tracking of cable-driven robots," IEEE Transactions on Industrial Informatics, vol. 16, no. 8, pp. 5234-5243, 2020.

[48] Y. Wang, F. Yan, J. Chen, and F. Ju, "A new adaptive timedelay control scheme for cable-driven manipulators," IEEE Transactions on Industrial Informatics, vol. 15, no. 6, pp. 3469-3481, 2019. 
[49] S. Park, R. Horowitz, and Y. Nam, "Trajectory-switching algorithm for a MEMS gyroscope," IEEE Transactions on Instrumentation and Measurement, vol. 56, no. 6, pp. 25612569, 2007.

[50] S. Ebrahimkhani, "Robust fractional order sliding mode control of doubly-fed induction generator (DFIG)-based wind turbines," ISA Transactions, vol. 63, pp. 343-354, 2016.

[51] S. Chen, H. Chiang, T. Liu, and C. Chang, "Precision motion control of permanent magnet linear synchronous motors using adaptive fuzzy fractional-order sliding-mode control," IEEE/ASME Transactions on Mechatronics, vol. 24, no. 2, pp. 741-752, 2019.

[52] W. Yan and J. Fei, "Adaptive control of MEMS gyroscope based on global terminal sliding mode controller," Mathematical Problem in Engineering, vol. 2013, Article ID 797626, 9 pages, 2013. 